\title{
Chancen für ein verfassungskonformes Asylbewerberleistungsgesetz
}

\begin{abstract}
Summary
In July 2012, the Federal Constitutional Court (Bundesverfassungsgericht, BVerfG) decided that the provision for basic benefits in the Asylum Seekers'Benefits Act (Asylbewerberleistungsgesetz, AsylbLG) was unconstitutional. The law-makers are now called upon to create a new law which is consistent with the constitution. In a first step, the following contribution describes how this special benefits regime under the AsylbLG was created and the jurisprudence on the issue. On the basis of the BVerfG's decision, but beyond that also the Basic Law's guidelines, an AsylbLG which is consistent with the constitution is then developed. This leads to an evaluation of currently discussed possibilities for amending the act. The contribution shows that the law-makers have great difficulties if they establish the benefits for asylum seekers or persons treated as such lower than for other people in Germany. A justification of such differences is more likely to succeed if the duration of this special treatment is short. It is also problematic that very different groups of people are classified as falling under the AsylbLG based on their residence permits. According to the author, a new AsylbLG which is consistent with the constitution must therefore be formulated much differently from the status quo. Whether law-makers will succeed at this seems questionable.
\end{abstract}

\section{Résumé}

Au mois de juillet 2012, la Cour constitutionnelle fédérale d'Allemagne a jugé inconstitutionnelle la disposition relative aux prestations de base issue de la loi sur les prestations sociales des demandeurs d'asile (AsylbLG). Le législateur est donc prié d'élaborer une nouvelle réglementation conforme à la Constitution. L'article ci-dessous met premièrement en lumière l'apparition du régime spécial de prestations sociales découlant de ladite loi et dessine une ébauche de la jurisprudence relative à ce sujet. Les directives de la Loi fondamentale relatives à une loi sur les prestations sociales des demandeurs d'asiles conforme à la Constitution sont ainsi développées en s'appuyant sur l'arrêt de la Cour constitutionnelle fédérale, mais également au-delà de ce cadre. En découle ensuite une appréciation des possibilités de modification envisagées à l'heure actuelle. $L$ 'article révèle que le législateur se heurte à des difficultés considérables lorsqu'il fixe des prestations sociales plus faibles pour les demandeurs d'asile et assimilés que pour les autres personnes résidant en Allemagne. Cette différence est plutôt justifiée lorsque ce traitement de faveur est accordé pour une durée réduite. Un autre problème consiste en ce que des groupes de personnes très divers sont subordonnés à la loi sur les prestations sociales des demandeurs d'asile, sur la base de leur titre de séjour. Une refonte conforme à la Constitution de la réglementation de ladite loi ne doit donc pas, de l'avis 
de l'auteure, se contenter d'un statu quo. Quant à savoir si le législateur y parviendra, cela reste encore à prouver.

\section{Ausgangslage}

\section{AsylbLG $G^{1}$}

Das Asylbewerberleistungsgesetz (AsylbLG) ist 1993 in Folge des sogenannten Asylkompromisses $^{2}$ in Kraft getreten. Es verfolgte das Ziel, die Sozialleistungen für Asylbewerberinnen und Asylbewerber gegenüber den Leistungen nach dem damaligen Bundessozialhilfegesetz (BSHG) sowohl im Umfang deutlich abzusenken als auch anstelle von Geldleistungen vorrangig Sachleistungen vorzusehen. Hintergrund waren schon in den 1980er Jahren entstandene Bestrebungen, Sozialleistungen für Asylbewerberinnen und Asylbewerber abzusenken. ${ }^{3}$ Die Zahl der Asylbewerber war seit dem Ende der 1980er Jahre bzw. seit 1990 massiv gestiegen, sodass der Gesetzgeber diese grundlegenden Reformen des Asylrechts für nötig hielt. In Bezug auf das AsylbLG stand das Ziel im Vordergrund, die Kosten für Aufnahme und Versorgung von Asylbewerbern zu verringern. ${ }^{4}$

Die Leistungsgestaltung und Leistungshöhe des AsylbLG ist seitdem unverändert. Sie ist im Grundsatz nach $\S 3$ AsylbLG festgelegt. Nach einer in $\S 2$ AsylbLG näher bestimmten Verweildauer in diesem Leistungsbezug nach $\S 3$ AsylbLG haben die Berechtigten einen Anspruch auf sogenannte Analogleistungen, also Leistungen, deren Umfang denen des SGB XII im Wesentlichen entspricht. Allerdings bleiben diese Personen systematisch dem AsylbLG zugeordnet.

\section{a) Veränderungen des Personenkreises}

Veränderungen hat jedoch der Kreis der Personen erfahren, die aus dem regulären Sozialleistungsbezug nach BSHG bzw. SGB II/SGB XII ausgeklammert und dem AsylbLG zugewiesen sind. Ursprünglich waren nur Asylbewerberinnen und Asylbewerber erfasst, deren Aufenthalt gemäß $§ 55$ AsylVerfG gestattet ist, sowie Personen, die (nach damaligem AuslG) vollziehbar ausreisepflichtig waren. Dieser Sonderung aus dem regulären Sozialhilferecht lag die Erwartung zugrunde, dass diese Personen nur für kurze Zeit in dem Sonderstatus bleiben würden. Entweder würde das Asylverfahren erfolgreich abgeschlossen und die Personen erhielten einen regulären ausländerrechtlichen Aufenthaltstitel mit der Folge, dass sie bei Hilfebedürftigkeit in die Sozialhilfe eingegliedert würden. Oder - mit deutlich überwiegender Wahrscheinlichkeit - das

1 Vgl. auch die Zusammenfassung wesentlicher Aspekte des AsylbLG in BVerfG, Urteil vom 18.7.2012 - BvL 10/10 und 1 BvL 2/11.

2 Wesentlicher Inhalt der Einigung zwischen CDU, CSU, FDP und SPD vom 6.12.1992 war die Streichung des alten Art. 16 Abs. 2 S. 2 GG und Schaffung des neuen Art. 16 a GG sowie Änderungen des AsylVerfG.

3 Beginnend mit dem 2. Haushaltsstrukturgesetz vom 22. Dezember 1981, BGB1 I S. 1523.

4 BT-Drs. 12/4451, S. 1 und 5. 
Asylgesuch würde abgelehnt und die Betroffenen würden alsbald Deutschland verlassen. ${ }^{5}$

1997 kamen als weitere Leistungsberechtigte des AsylbLG Ausländerinnen und Ausländer hinzu, die - so die Begründung des Gesetzgebers ${ }^{6}$ - keinen verfestigten Aufenthaltsstatus hatten und sich daher typischerweise vorübergehend in Deutschland aufhielten. Dies waren insbesondere Kriegs- und Bürgerkriegsflüchtlinge. ${ }^{7}$ Bei der Reform des Ausländerrechts durch das Zuwanderungsgesetz 2004 und dessen Novellierung 2007 wurde diese Konzeption dadurch fortgeführt, dass Inhaber verschiedener humanitärer Aufenthaltstitel dem AsylbLG zugewiesen wurden. Sie lassen sich in zwei systematische Gruppen aufteilen: Zum einen handelt es sich um Kriegsflüchtlinge mit einem Aufenthaltstitel nach $\S 23$ Abs. 1 oder $\S 24$ AufenthG. Zum anderen geht es um Personen mit Aufenthaltstiteln gemäß $\S 25$ Abs. 4 bis Abs. 5 AufenthG ${ }^{8}$ und Geduldete gemäß $\S 60$ a AufenthG - sämtlich Personen, die grundsätzlich ausreisepflichtig sind, bei denen die Ausreisepflicht aber aus unterschiedlichen Gründen nicht vollzogen werden kann.

Angesichts der Gesamtkonzeption des AufenthG und in Anbetracht der tatsächlichen Aufenthaltsdauer dieser Personengruppen muss allerdings die gesetzgeberische Konzeption in zweierlei Hinsicht präzisiert werden.

Die Umschreibung der dem AsylbLG zugewiesenen Aufenthaltstitel als ,nicht verfestigter ausländerrechtlicher Status“ erfasst nicht alle Aufenthaltstitel für einen kurzzeitigen Aufenthalt. Insbesondere der Aufenthalt $\mathrm{zu}$ Ausbildungszwecken ( $\$ 16$ AufenthG), für befristete Beschäftigung (vgl. etwa für Saisonbeschäftigung $\S 18,39$ AufenthG i.V.m. $\S 18$ BeschV) oder die sog. Schengenvisa (§ 6 Abs. 1 Nr. 1 AufenthG) sind ebenfalls nicht auf Verfestigung des ausländerrechtlichen Status gerichtet. Sie unterscheiden sich von den Personengruppen des AsylbLG aber dadurch, dass ihr Aufenthalt grundsätzlich bei Hilfebedürftigkeit beendet werden kann (s. $\S 5$ Abs. $1 \mathrm{Nr} .1$ AufenthG). Die Personengruppen des AsylbLG behalten hingegen auch ohne Sicherung ihres Lebensunterhalts ihren Aufenthaltsstatus (vgl. § 5 Abs. 3 AufenthG) oder jedenfalls ist ihre Ausreise nicht vollziehbar. Es kommt also bei der Zuweisung zum AsylbLG

5 BT-Drs. 12/441, S. 5: „Dadurch können die Leistungen gegenüber der Sozialhilfe, die vom Individualisierungsgrundsatz ausgeht und ein existentiell gesichertes und sozial integriertes Leben der Leistungsberechtigten ,auf eigenen Füßen“ in der Bundesrepublik Deutschland zum Ziel hat, vereinfacht und auf die Bedürfnisse eines hier in aller Regel nur kurzen, vorübergehenden Aufenthaltes abgestellt werden. [...] Zum anderen handelt es sich um abgelehnte Asylbewerber, die nicht aus anderen anzuerkennenden Gründen längerfristig geduldet werden oder bei denen die Duldung auf Gründen beruht, die sie selbst zu vertreten haben." (Hervorhebungen A.W.).

6 BT-Drs. 13/2746, S. $11=$ BT-Drs 13/3475, S. 2.

7 Vgl. zur parlamentarischen Auseinandersetzung hierüber BT-Prot. 13/172, S. 15524.

8 Die Herausnahme der Verlängerung gemäß $\S 25$ Abs. 4 S. 2 AufenthG wird hier nicht weiter vertieft. Die Konzeption des AsylbLG ist, dass Personen, bei denen der humanitäre Aufenthaltstitel gemäß $\S 25$ Abs. 4 S. 2 AufenthG verlängert wurde, nicht mehr unter das AsylbLG fallen, sondern den Regelsystemen des SGB II/SGB XII zugewiesen sind, vgl. Deibel, ZAR 2011, 178, 179. Ausländerrechtlich ist jedoch noch nicht abschließend geklärt, in welchen Fällen eine Verlängerung auf $\S 25$ Abs. 4 S. 2 AufenthG und nicht anderen Bestimmungen des AufenthG beruht, vgl. Deibel, ebenda. 
nicht darauf an, ob der Aufenthaltstitel verfestigt ist, sondern darauf, dass er - obwohl er nicht verfestigt ist - nicht beendet werden kann.

Auch die Beschreibung, dass es sich um ,typischerweise vorübergehende Aufenthalte" handelt, bedarf der Präzisierung. Aus der Unmöglichkeit, den Aufenthalt der betroffenen Personengruppen zu beenden, folgt geradezu zwangsläufig, dass der tatsächliche Aufenthalt keineswegs immer kurzfristig und vorübergehend ist. Typischerweise müssen sich Veränderungen im Herkunftsstaat oder in der persönlichen Situation der Betroffenen ergeben, bevor der Aufenthalt tatsächlich beendet wird. Solche Veränderungen führen dabei zugleich auch zu einer Änderung des Aufenthaltstitels selbst (vgl. $\S 26$ Abs. 2 AufenthG), so dass die Zuordnung zum AsylbLG, die ja am Aufenthaltstitel ansetzt, schon dadurch endet. Oder sie erfolgt aufgrund von $\S 1$ Abs. 1 Nr. 5 AsylbLG, wenn ein Ausländer gar keinen Aufenthaltstitel hat und vollziehbar ausreisepflichtig ist. Tatsächlich halten sich viele der betroffenen Personen auch mehrere Jahre, teilweise schon ganze Generationen, in Deutschland auf. ${ }^{9}$ Wäre dies nicht faktisch der Fall, hätte es der geschilderten Änderungen des AsylbLG auch nicht bedurft oder zumindest wären damit keine relevanten Einsparungen für die Sozialhilfeträger verbunden gewesen. ${ }^{10}$ Grund für die Zuordnung der betroffenen Personen zum AsylbLG ist also nicht die tatsächliche Erwartung, dass ihr Aufenthalt vorübergehend sein wird, sondern vielmehr, dass aufgrund tatsächlicher Begebenheiten der politische Wunsch nicht realistisch ist, dass der Aufenthalt vorübergehend sein soll.

Die beiden Merkmale, die als Grund für die Zuordnung zum AsylbLG angegeben werden - der nicht verfestigte Status und der vorübergehende Aufenthalt - sind also letztlich eher kontrafaktische politisch-normative Erwartungen.

Etwas anderes gilt nur für die Personengruppe, die vollziehbar ausreisepflichtig ist ( 1 Abs. 1 Nr. 5 AsylbLG). In diesen Fällen, in denen ein Aufenthaltstitel gar nicht besteht und auch keine Abschiebungshindernisse bestehen, dürfte der Norm die Erwartung zugrunde liegen, dass die Betroffenen nur noch eine begrenzte Aufenthaltszeit vor sich haben.

Zusammenfassend lässt sich festhalten, dass für die Personengruppen, die dem AsylbLG zugewiesen sind, sehr unterschiedliche Aufenthaltsprognosen bestehen. Für Asylbewerber ist diese Prognose zunächst - in der ersten Aufenthaltsphase, während der das Asylverfahren stattfindet - offen. Bei den in $\S 1$ Abs. 1 Nr. 3 AsylbLG genannten Aufenthaltstiteln stehen der politischer Wunsch der Aufenthaltsbeendigung und die Wirklichkeit, in der aus verschiedenen Gründen Hindernisse bestehen, einander gegenüber. Die Prognose einer baldige Aufenthaltsbeendigung liegt letztlich nur $\S 1$ Abs. 1 Nr. 5 AsylbLG zugrunde.

\section{b) Veränderungen der Verweildauer im abgesenkten Bezug}

Nicht nur der Personenkreis der Leistungsberechtigten hat sich erweitert. Auch die Verweildauer im ,eigentlichen“ AsylbLG-Bezug - im Bezug der Leistungen nach $\S 3$ AsylbLG - wurde verlängert, indem der Zugang zu den Analogleistungen nach $§ 2$

9 Auch das Bundesverfassungsgericht hat dies aufgegriffenen und differenziert in seinem Urteil dargestellt: BVerfG, Urteil vom 18.7.2012 - 1 BvL 10/10 und 1 BvL 2/11, Rn. 15.

10 Zum erwarteten Sparvolumen für Länder und Kommunen vgl. BT-Prot. 13/172, S. 15523. 
AsylbLG immer weiter heraufgesetzt wurde. Diese Verlängerung erfolgte auf zweierlei Weise. Zum einen wurde die Zeitspanne heraufgesetzt, nach der ein Wechsel zu den Analogleistungen möglich wird. Zum anderen wurde der Beginn dieser Vorbezugszeit verändert.

Ursprünglich waren Analogleistungen für die Leistungsberechtigten vorgesehen, über deren Asylantrag 12 Monate nach Antragsstellung noch nicht entschieden war. Im Ergebnis erhielten damit alle Personen nach spätestens einem Jahr, in manchen Fällen auch früher, Leistungen in Höhe der Sozialhilfe.

Mit dem Ersten Gesetz zur Änderung des AsylbLG ${ }^{11}$ wurden 1997 die Leistungsberechtigten erst dann bessergestellt, wenn sie über eine Dauer von insgesamt 36 Monaten Leistungen nach $\S 3$ AsylbLG erhalten hatten. Der Gesetzentwurf hatte zunächst nur Personen mit einer Duldung ( $\$ 55$ AuslG a.F.) in den persönlichen Anwendungsbereich einbeziehen wollen. Dabei sollten die Geduldeten höchstens zwei Jahre ab Duldungserteilung im abgesenkten Bezug verbleiben. Dies begründete der Gesetzentwurf damit, dass zunächst bei Geduldeten davon auszugehen sei, dass sie Deutschland auch verlassen würden. Nach zwei Jahren sei aber diese Prognose nicht mehr aufrecht zu erhalten. ${ }^{12}$ Nach einjähriger Beratung im Vermittlungsausschuss erhielt die Neuregelung die genannte, gegenüber dem Entwurf massiv verschärfte, Gestalt. Neben den Einsparinteressen der Länder ${ }^{13}$ sind hierfür keine sachlichen Erwägungen erkennbar. ${ }^{14} \mathrm{Im}$ Ergebnis können dadurch Personen, die dem AsylbLG zugewiesen sind, auch nach langjährigem Aufenthalt in Deutschland nur abgesenkte Leistungen erhalten. Dies gilt insbesondere dann, wenn die Betroffenen nicht ununterbrochen auf Leistungen nach dem AsylbLG angewiesen sind, sondern ihren Lebensunterhalt zwischenzeitlich auch anders sichern können. Bei Unterbrechungen gilt nach der Rechtsprechung des Bundessozialgericht grundsätzlich eine Addition der Bezugszeiträume, solange ein und derselbe Leistungsfall vorliegt. ${ }^{15}$

Mit dem Zuwanderungsgesetz $2004^{16}$ wurde als weitere Voraussetzung für die Leistungsanhebung verlangt, dass die Berechtigten die Dauer ihres Aufenthalts nicht rechtsmissbräuchlich selbst beeinflusst hatten. In der Literatur wurde als Gesetzgebungsziel angegeben, durch die abgesenkten Leistungen sowie die restriktive Gestaltung des Übergangs zu den Analogleistungen sollte Ausländern der Anreiz für die Einreise und den weiteren Verbleib in Deutschland genommen werden. ${ }^{17}$

11 BGBl. I 1997, S. 1130.

12 BT-Drs. 13/2746, S. 5, 15.

13 BT-Prot. 13/172, S. 15523.

14 Vgl. auch zur Gesetzesgenese BSGE 101, 49, Rn. $20 \mathrm{f}$.

15 Zu den Einzelheiten vgl. BSGE 101, 49, 54; 103, 28, 30 f. m.w.Nw.

16 BGBl I 2004, 1950, Art. 8.

17 Hohm, AsylbLG (Stand 2007), § 2 Rn. 86; zitiert in BSGE 101, 49, 57; in anderem Kontext zitiert das BSG die Gesetzesbegründung von 1993 dafür, dass keine Bleibeanreize gesetzt werden sollten: BSGE 102, 60, Rn. 25. Dieses Motiv bezieht der Gesetzgeber allerdings nur auf die Personengruppe abgelehnter und deshalb vollziehbar ausreisepflichtiger Asylbewerber, die nicht aus anderen Gründen zu dulden sind: BT-Drs. 12/4451, S. 5. Dass Anreize, nicht zu Bleiben, auch bei Personen gesetzt werden sollen, die einen humanitären Aufenthaltstitel oder eine Duldung besitzen, war vom Gesetzgeber 1993 so nicht vorgesehen. 
Schließlich wurde 2007 die erforderliche Bezugsdauer auf 48 Monate erhöht. ${ }^{18}$ Zur Begründung wurde auf eine Angleichung zur Regelung des $\S 10$ BeschVerfV verwiesen, der eine Zustimmung der Bundesagentur für Arbeit zur Beschäftigung auch ohne Vorrangprüfung ${ }^{19}$ nach Ablauf von vier Jahren Aufenthalt in Deutschland vorsieht. ${ }^{20}$ Dass die 48-monatige Vorbezugszeit nach $\S 2$ AsylbLG gerade keinen Gleichlauf mit einem vierjährigen Aufenthalt darstellt, wurde schlicht verkannt oder ignoriert. ${ }^{21}$

Will man den Charakter der Leistungsunterscheidung zwischen $\S 3$ und $\S 2$ AsylbLG zusammenfassen, so lässt sich sagen, dass der ursprünglichen Regelung der Gedanke zugrunde lag, dass in einer Anfangszeit in Deutschland - insbesondere unter den weiteren Bedingungen der (Erst-)Aufnahmen von Asylbewerbern und Flüchtlingen - ein anderer und gegenüber „normalen“ Sozialhilfeleistungen abgesenkter Bedarf angenommen wurde. Die Änderungen haben diesen Begründungsstrang allerdings ganz in den Hintergrund treten lassen. Eine tragfähige Begründung ist - neben fiskalischen Interessen - auch in den Gesetzentwürfen hierfür nicht erkennbar.

\section{Relevante Änderungen anderer Regelungen}

Um die Regelungen des AsylbLG einordnen zu können, sind neben diesen Änderungen des AsylbLG zudem die Veränderungen der ,,angrenzenden“ Rechtsgebiete mit in den Blick zu nehmen. Hierzu gehören neben dem bereits angesprochenen Ausländerrecht das Beschäftigungsrecht, gemeint sind damit die Regelungen, die Ausländern die Beschäftigung in Deutschland erlauben, sowie die Reform der Sozialhilfe bzw. der Grundsicherung.

\section{a) Beschäftigungserlaubnis}

Als 1993 das AsylbLG geschaffen wurde, waren die Beschäftigungsmöglichkeiten für Ausländer in Deutschland beschränkter als heute. Das Ausländer- und Beschäftigungsrecht ging grundsätzlich davon aus, dass Ausländer nur mit einem Aufenthaltstitel zu Erwerbszwecken erwerbstätig sein durften (vgl. § 284 ff. SGB III a.F.). Ein Aufenthaltstitel aus anderen Gründen, insbesondere die Gestattung während eines Asylverfahrens, der Flüchtlingsstatus oder die Duldung erlaubten grundsätzlich keine Beschäf-

18 BGBl. I 2007, 1970.

19 Hierzu s. auch unten.

20 BT-Drs. 16/5065 S. 232.

21 Wörtlich heißt es in der Gesetzesbegründung: „Damit wird eine einheitliche Stufung nach vier Jahren eingeführt. [...] Die Entscheidung über den Beginn der sozialen Einbindung und damit über die Gewährung der höheren Leistungen nach dem Sozialgesetzbuch, die für die Integration in hiesige Lebensverhältnisse zu gewähren sind, hängt von dem Grad der zeitlichen Verfestigung des Aufenthalts ab." (Hervorhebung A.W.) - Nach Auffassung des BSG hat der Gesetzgeber aber gleichwohl seinen fortbestehenden Willen, die Vorbezugszeit erst mit Bezugsbeginn anfangen zu lassen, zum Ausdruck gebracht, BSGE 101, 49, 57, s. dazu auch unten. 
tigung. Allerdings waren seit 1998 gemäß $§ 284$ Abs. 5 SGB III a.F. i.V.m. $\S 5$ $\mathrm{ArgV}^{22}$ Genehmigungen im Ausnahmefall möglich.

Mit dem Zuwanderungsgesetz wurden die Beschäftigungsrestriktionen gelockert. Zwar galt und gilt weiterhin der Grundsatz, dass der Aufenthaltstitel ausdrücklich zur Erwerbstätigkeit berechtigen muss ( $\$ 4$ Abs. 3 AufenthG). Aber insbesondere die verschiedenen humanitären Aufenthaltstitel sind nicht mehr grundsätzlich beschäftigungsfeindlich. Vielmehr ist die Zulassung zum Arbeitsmarkt grundsätzlich bei jedem Aufenthaltstitel möglich, unterliegt dabei jedoch der Zustimmung durch die Bundesagentur für Arbeit ( $\S 4$ Abs. 2 S. 2, 39 ff. AufenthG). Seit 2005 gilt, dass alle Ausländer, auch Asylbewerber, deren Aufenthalt nach $\S 55$ AsylVerfG gestattet ist, und auch Geduldete nach einem Jahr Aufenthalt in Deutschland zum Arbeitsmarkt zugelassen werden können (§ 61 Abs. 2 AsylVerfG, $\S \S 39$ ff. AufenthG). Soweit nicht für bestimmte Branchen oder Berufe Freistellungen vom Zustimmungserfordernis vorgesehen sind (BeschV und BeschVerfV), ist die Vorrangprüfung gem. § 39 Abs. 2 S. 1 AufenthG entscheidend.

Im Ergebnis können damit Leistungsberechtigte nach dem AsylbLG nach einem Jahr Aufenthalt in Deutschland eine Beschäftigungserlaubnis erhalten, wenn auf dem Arbeitsmarkt keine bevorrechtigten Arbeitnehmer, insbesondere Deutsche und Unionsbürger, aber auch Personen mit Niederlassungs- oder zur Erwerbstätigkeit berechtigender Aufenthaltserlaubnis zur Verfügung stehen. Allein der Aufenthaltsstatus führt also nicht mehr wie 1993 gewissermaßen zwingend zur Hilfebedürftigkeit. Umgekehrt führt aber der Verlust einer Erwerbstätigkeit und daraus folgende Hilfebedürftigkeit dazu, dass sich der Bezug der Leistungen nach $\S 3$ AsylbLG an eine unter Umständen auch mehrjährige Erwerbstätigkeit in Deutschland anschließen kann. Wenn man annehmen will, dass bis 1993 und 1997 Aufenthaltsstatus und Bezug von Leistungen nach dem AsylbLG jedenfalls faktisch oft miteinander gekoppelt sind, so gilt dies heute nicht mehr. Vielmehr verliefen integrationsfreundliche Lockerungen des Beschäftigungsrechts mit Verschärfungen des AsylbLG und der damit verbundenen Schlechterstellung gegenüber anderen Hilfebedürftigen bemerkenswert gleichzeitig.

\section{b) Änderungen des Grundsicherungsrechts}

Nicht nur das Ausländerrecht hat seit 1993 grundlegende Änderungen erfahren. Auch das Recht der Sozialhilfe hat einen Paradigmenwechsel erlebt. Bis zum 31.12.2004 erfolgte die Sicherstellung des Lebensunterhalts erwerbsfähiger Personen durch die Arbeitslosenhilfe nach $\S \S 190$ ff. SGB III a.F. auf der einen und der Sozialhilfe nach BSHG auf der anderen Seite. Mit den sogenannten Hartz-Reformen ${ }^{23}$ wurde die bisherige Sozialhilfe in die Grundsicherung für Erwerbsfähige einerseits (SGB II) und die Grundsicherung für nicht Erwerbsfähige andererseits (SGB XII) differenziert.

Für die Bewertung des AsylbLG sind dabei neue Zuordnungen der Kosten zwischen Bund auf der einen und Ländern bzw. Kommunen auf der anderen Seite relevant. Seit 2005 trägt der Bund die Kosten des Regelbedarfs für Erwerbsfähige nach dem SGB II, soweit die Bundesagentur für Arbeit zuständig ist, § 46 Abs. 1 S. 1 SGB II. Die Kom-

22 BGBl. I 1998, S. 2899.

23 Insbesondere das Vierte Gesetz für moderne Dienstleistungen am Arbeitsmarkt vom 24.12.2003, BGBl. I S. 2954. 
munen bzw. Länder tragen damit „nur“ noch den Regelbedarf für Nicht-Erwerbsfähige nach dem SGB XII sowie die Kosten für Unterkunft und Heizung für alle Bedürftigen, entsprechend ihrer originären Zuständigkeit, § 22 SGB II und § 3 SGB XII (auf weitere Leistungsarten wird hier nicht eingegangen). Für die Kostenzuordnung im Bundesstaat ist damit die Eigenschaft der Erwerbsfähigkeit i.S.d. § 8 SGB II wesentlich.

Dies wird auch an der Neufassung des $\S 8$ Abs. 2 SGB II ${ }^{24}$ deutlich. Der Gesetzgeber stellt mit dem neuen $\S 8$ Abs. 2 S. 2 SGB II klar, dass Ausländerinnen und Ausländer bereits dann als erwerbsfähig gelten, wenn die rechtliche Möglichkeit besteht, dass ihnen eine Beschäftigung gemäß $§ 39$ AufenthG erlaubt wird. Es genügt also die abstrakte Möglichkeit, dass das Beschäftigungserlaubnisgesuch bei einer Vorrangprüfung Erfolg haben kann. Im Ergebnis sind damit Ausländerinnen und Ausländer, die in den Anwendungsbereich des SGB II fallen, nach einem Jahr Aufenthalt in Deutschland erwerbsfähig. Die Kosten für ihren Regelbedarf trägt daher der Bund. Die Zuordnung zum AsylbLG verhindert diese Zuordnung für den betroffenen Personenkreis. ${ }^{25}$ Die Kosten für den Regelbedarf der Ausländerinnen und Ausländer, die dem AsylbLG zugewiesen sind, müssen - entsprechend der traditionellen Kostenlast der Sozialhilfe - von den Kommunen bzw. Ländern getragen werden. An der Kostenzuweisung entsprechend der Erwerbsfähigkeit, wie sie die neue Systematik des SGB II/SGB XII und speziell $\S 8$ Abs. 2 SGB II vornimmt, haben diese Personengruppen nicht teil.

\section{Einschlägige höchstrichterliche Rechtsprechung}

\section{a) BVerfG-Urteil vom 9.2.2010 und Reform des SGB II}

Von unmittelbarer Bedeutung für die anstehende Neuregelung des AsylbLG ist das Urteil des Bundesverfassungsgerichts vom 9.2.2010 zur Verfassungswidrigkeit der Regelsätze nach dem SGB II. ${ }^{26}$ Denn das Bundesverfassungsgericht hat aus Art. 1 Abs. 1 GG i.V.m. dem Sozialstaatsprinzip einen verfassungsrechtlichen Anspruch auf Gewährung des menschenwürdigen Existenzminimums abgeleitet. Materiellrechtlich umfasst dies sowohl die physische Existenz, zu der Nahrung, Kleidung, Hausrat, Unterkunft, Heizung, Hygiene und Gesundheit zählen, als auch die Sicherung der Möglichkeit zur Pflege zwischenmenschlicher Beziehungen und zu einem Mindestmaß an Teilhabe am gesellschaftlichen, kulturellen und politischen Leben. ${ }^{27}$ Dieser Anspruch ist dem Grunde nach unverfügbar. Sein Inhalt ist aber durch den Gesetzgeber anhand der vorherrschenden gesellschaftlichen Anschauungen über das für ein menschenwürdiges Dasein Erforderliche, der jeweiligen Lebenssituation des Bedürftigen und den jeweiligen wirtschaftlichen und technischen Gegebenheiten zu konkretisieren und stetig zu aktualisieren. ${ }^{28}$ Der Gesetzgeber muss bei dieser Konkretisierung des grundrechtlichen Anspruchs alle existenznotwendigen Aufwendungen folgerichtig in einem transparenten und sachgerechten Verfahren nahe dem tatsächlichen Bedarf, also realitätsgerecht, be-

24 Art. 2 des EGRBEG, BGBl. 2011 I S 453, $459 \mathrm{f}$.

25 Vgl. BSGE 102, 60, $63 \mathrm{f}$.

26 BVerfGE $125,175$.

27 BVerfGE 125, 175, 223.

28 BVerfGE 125, 175, $222 \mathrm{f}$. 
messen. Welcher Ermittlungs- und Berechnungsmethode er sich dabei bedient, ist nicht vom Grundgesetz vorgeschrieben, sondern unterliegt nur den Voraussetzungen der Tauglichkeit und Sachgerechtigkeit. Abweichungen von der gewählten Methode bedürfen allerdings der sachlichen Rechtfertigung. ${ }^{29}$ Außerdem ist der Gesetzgeber verpflichtet, Vorkehrungen zu treffen, um auf Änderungen der wirtschaftlichen Rahmenbedingungen wie Preissteigerungen oder Erhöhungen von Verbrauchssteuern zeitnah reagieren zu können. ${ }^{30}$ Sind diese Voraussetzungen erfüllt, beschränkt sich die gerichtliche Kontrolle darauf, ob das gefundene Ergebnis evident unzureichend ist. ${ }^{31}$

Die Anwendung dieser Grundsätze auf die damaligen Regelleistungen des $\S 20$ Abs. 2 SGB II führte zur Feststellung ihrer Verfassungswidrigkeit. Zwar war die Höhe der Regelsätze selbst nicht evident unzureichend, aber der Gesetzgeber hatte die verfassungsrechtlichen Anforderungen zur Ermittlung der Regelsatzhöhe missachtet. Das Berechnungsmodell beruhte zwar mit der Einkommens- und Verbrauchsstichprobe 1998 auf einer hinreichend sorgfältig ermittelten Tatsachengrundlage und daraus abgeleitet auch grundsätzlich auf einem nachvollziehbaren Verfahren. Aber der Gesetzgeber war von diesem Modell der Bedarfsermittlung ohne erkennbaren sachlichen Grund abgewichen. ${ }^{32}$

Der Gesetzgeber hat die Regelbedarfsermittlung im Jahr 2011 mit dem Gesetz zur Ermittlung von Regelbedarfen und zur Änderung des Zweiten und Zwölften Buches des Sozialgesetzbuch (EGRBEG) ${ }^{33}$ neu vorgenommen, um den genannten verfassungsrechtlichen Anforderungen zu genügen.

In Folge dieses Urteils wurde alsbald auch die Frage aufgeworfen, ob bei Zugrundelegung der genannten Maßstäbe die Leistungsbemessung des $§ 3$ AsylbLG ebenfalls verfassungswidrig sei. ${ }^{34}$

\section{b) Einschlägige Rechtsprechung der Sozialgerichte zu $\S \S 2$ und 3 AsylbLG}

Schließlich sei auf aktuelle Rechtsprechung der Sozialgerichte zum AsylbLG hingewiesen.

Die lange Vorbezugsdauer - also die Frist, in der Leistungen nach $\S 3$ AsylbLG bezogen worden sein müssen, bevor sogenannte Analogleistungen nach $\S 2$ AsylbLG gewährt werden - und insbesondere ihre Verlängerung durch Art. 6 Abs. 2 des Gesetzes zur Umsetzung aufenthalts- und asylrechtlicher Richtlinien der Europäischen Union vom 19.8.2007 $7^{35}$ von 36 auf 48 Monate hat zu vielen Rechtsstreitigkeiten geführt. Unterinstanzen hielten es auch aus verfassungsrechtlichen Gründen für geboten, auf die

29 BVerfGE 125, 175, 225.

30 BVerfGE 125, 175, 225.

31 BVerfGE 125, 175, $225 \mathrm{f}$.

32 BVerfGE 125, 175, $238 \mathrm{ff}$.

33 BGB1. I 2011, S. 453.

34 Vgl. Classen/Kanalan, info also 2010, 243; Hädrich, ZAR, 2010, 227; Janda/Wilksch, SGb 2012, 565; Kingreen, NVwZ 2012, 558; Rothkegel, ZAR 2010, 373; Görisch, NZS 2011, 646; Mahler/Follmar-Otto, ZAR 2011, 378.

35 BGBl. I 2007, S. 1970. 
Vorbezugszeit auch andere Leistungen als die des $\S 3$ AsylbLG anzurechnen. ${ }^{36}$ Dem hat das Bundessozialgericht widersprochen. ${ }^{37}$ Es sah für eine solche Auslegung keinen Spielraum, da der Wille des Gesetzgebers eindeutig sei. Allein Leistungen nach $\S 3$ AsylbLG könnten die Vorbezugszeiten erfüllen. Auch bestehen nach Ansicht des Bundessozialgerichts hiergegen keine verfassungsrechtlichen Bedenken für Personen, deren Leistungen „rückgestuft“ wurden. Weder werde Art. 3 Abs. 1 GG verletzt, da alle Leistungsberechtigten gleich gestellt würden, noch liege ein Verstoß gegen das Rückwirkungsverbot vor.

Ebenso hat das Bundessozialgericht die Zuweisung der gesetzlich vorgesehenen Personengruppen zum AsylbLG anstatt zum SGB II für verfassungskonform gehalten. ${ }^{38}$ Der sachliche Differenzierungsgrund für die Zuweisung zu einem alternativen Leistungssystem bestehe darin, dass der Gesetzgeber den Anreiz zur Einreise bzw. zum Bleiben verringern wolle. Dies sei für die Personengruppen mit den Aufenthaltstiteln des $\S 1$ Abs. 1 Nr. 3 AsylbLG ein zulässiges Differenzierungskriterium. Außerdem ermöglichten die Analogleistungen des $\S 2$ AsylbLG die verfassungsrechtlich gebotene soziale Integration in den Fällen, in denen sich der Aufenthalt doch verfestige, unter Achtung des gesetzgeberischen Anliegens, keine Bleibeanreize zu setzen.

In Folge dieser letztinstanzlichen Rechtsprechung hat sodann das Landessozialgericht NRW in zwei Verfahren die Regelsatzbestimmung des $\S 3$ AsylbLG dem Bundesverfassungsgericht vorgelegt. ${ }^{39}$ In den Verfahren begehrten Personen, die dem AsylbLG zugeordnet waren, die höheren Analogleistungen gemäß $§ 2$ AsylbLG. Die Kritik der Beschwerdeführer richtete sich dabei in erster Linie gegen die Heraufsetzung der Vorbezugszeit, also das Problem, dass das BSG soeben entschieden hatte. Da sich das LSG aus den genannten Gründen nicht in der Lage sah, die Zugangsvoraussetzungen für die Analogleistungen nach $\S 2$ AsylbLG so auszulegen, dass die Kläger die begehrten Leistungen erhielten, kam es zu der Überzeugung, dass $\S 3$ AsylbLG wegen unzureichender Höhe der Regelleistung verfassungswidrig sei. Es legte daher diese Norm dem Bundesverfassungsgericht vor.

\section{c) Urteil des BVerfG vom 18.7.2012 zur Verfassungswidrigkeit des AsylbLG}

Auf diese Vorlage hin hat das Bundesverfassungsgericht mit Urteil vom 18.7.2012 33 AsylbLG als verfassungswidrig verworfen. ${ }^{40}$ Es sah bereits die Höhe der Regelleistungen nach $\S 3$ AsylbLG als evident unzureichend an, so dass dadurch gegen die grundrechtliche Garantie eines menschenwürdigen Existenzminimums verstoßen werde. Außerdem könne die Leistungshöhe weder nachvollziehbar berechnet noch transparent und nachvollziehbar aus tatsächlichen Grundlagen heraus ermittelt werden. Auch aus diesem Grund liege ein Verstoß gegen Art. 1 Abs. 1 GG i.V.m. dem Sozialstaatsprinzip vor.

36 Vgl. etwa LSG Hessen, Beschluss vom 21.3.2007 - L 7 AY 14/06 ER, vgl. LSG Niedersachsen-Bremen, Beschluss vom 19.5.2008 - S 3 B 168/08, S 3 S 169/08, Rn. 15 m.w.Nw.

37 BSGE 101, 49; vgl. auch Hohm, NVwZ 2008, 33, 36.

38 BSGE 102, 60.

39 LSG NRW, Vorlagebeschluss vom 26.7.2010 - L 20 AY 13/09, Vorlagebeschluss vom 22.10.2010 - L 20 AY 1/09.

40 BVerfG, Urteil vom 18.7.2012 - 1 BvL 10/10 und 1 BvL 2/11. 
Das Bundesverfassungsgericht gab dem Gesetzgeber auf, unverzüglich eine verfassungskonforme Neuregelung zu schaffen. Für die Übergangszeit ordnete es - zur Realisierung der grundrechtlichen Garantie eines menschenwürdigen Existenzminimums eine auch in der Leistungshöhe genau bezifferte Regelleistung anstelle der gesetzlichen Leistungshöhe des $\S 3$ AsylbLG an.

Die wichtigsten Aussagen des Urteils für die Neugestaltung des AsylbLG sind:

Aus Art. 1 Abs. 1 GG i.V.m. dem Sozialstaatsprinzip folgt ein unmittelbar verfassungsrechtlicher Leistungsanspruch auf Gewährleistung eines menschenwürdigen Existenzminimums. Der Gesetzgeber muss dieser Gewährleistung durch Normierung eines gesetzlichen Anspruchs Rechnung tragen. Die dabei erforderliche konkrete Bestimmung des Leistungsanspruchs hängt von den gesellschaftlichen Anschauungen über das für ein menschenwürdiges Dasein Erforderliche, der konkreten Lebenssituation der Hilfebedürftigen sowie den jeweiligen wirtschaftlichen und technischen Gegebenheiten ab. Die Ausgestaltung, die der Gesetzgeber zu finden hat, muss folgerichtig in einem inhaltlich transparenten und sachgerechten Verfahren nach dem tatsächlichen und jeweils aktuellen Bedarf, also realitätsgerecht bemessen, begründet werden können (vgl. ebenso BVerfGE 125, 175).

Dabei geht es nicht um spezifische Verfahrenspflichten des Gesetzgebers, sondern entscheidend ist, dass sich der Rechtsanspruch auf existenzsichernde Leistungen durch realitätsgerechte, schlüssige Berechnungen sachlich differenziert begründen lässt (Rn. 96).

Eine bestimmte Methode ist dem Gesetzgeber nicht vorgegeben. Werden aber hinsichtlich bestimmter Personengruppen unterschiedliche Methoden gewählt, so muss dies sachlich zu rechtfertigen sein. Eine Methode, die Bedarfe von vornherein ausblendet, obwohl diese ansonsten als existenzsichernd anerkannt worden sind, darf nicht gewählt werden (Rn. 97).

Die Leistungsbemessung ist fortwährend $\mathrm{zu}$ überprüfen und weiterzuentwickeln (Rn. 98).

Eine Differenzierung bestimmter Personengruppen pauschal nach dem Aufenthaltsstatus ist nicht zulässig. Erforderlich ist vielmehr, dass der Bedarf dieser Personengruppen von dem anderer Bedürftiger signifikant abweicht. Diese Abweichung muss folgerichtig in einem inhaltlich transparenten Verfahren anhand des tatsächlichen Bedarfs gerade dieser Gruppe belegt werden können (Rn. 99).

Ob der Bedarf von Menschen mit vorübergehendem Aufenthaltsrecht abweichend vom Bedarf anderer Hilfebedürftiger bestimmt werden kann, hängt allein davon $a b$, ob wegen eines kurzfristigen Aufenthalts konkrete Minderbedarfe festgestellt und bemessen werden können (Rn. 100).

Ist dies der Fall, muss die gesetzliche Umschreibung der gesondert behandelten Personengruppe hinreichend zuverlässig tatsächlich nur diejenigen erfassen, die sich regelmäßig nur kurzfristig in Deutschland aufhalten. Der Aufenthaltsstatus ist zu Beginn des Aufenthalts ein - wenn auch allein kein hinreichender - Indikator für eine solche Prognose. Die Einbindung des Aufenthaltsstatus in die tatsächlichen Verhältnisse ist dabei aber stets zu berücksichtigen (Rn. 101).

Eine Rechtfertigung für die gesetzliche Anordnung eines geminderten Bedarfs für Kurzaufenthalte scheitert jedenfalls dann, wenn unabhängig vom jeweiligen Aufenthaltsstatus und ohne Rücksicht auf die Berechtigung einer ursprünglich gegenteiligen 
Prognose der tatsächliche Aufenthalt die Spanne eines Kurzaufenthalts deutlich überschritten hat. Verfassungsrechtlich geboten ist für diese Fälle ein gesetzlich geregelter zeitnaher Übergang zu den Normalfällen (Rn. 102).

Die verfassungsrechtlichen Vorwürfe an die gegenwärtige Regelung sind:

Die Leistungshöhe ist evident unzureichend (Rn. 107-116).

Es sind keine belastbaren Bemessungsgrundlagen für die gesetzliche Leistungshöhe erkennbar (Rn. 117).

Es gibt keine plausiblen Belege für die dem AsylbLG zugrunde liegende Vermutung, dass sich die Berechtigten nur kurzzeitig in Deutschland aufhalten (Rn. 118-119).

Eine kurze Aufenthaltsdauer oder Aufenthaltsperspektive ändert nichts daran, dass bereits ab Beginn des Aufenthalts in Deutschland eine menschenwürdige Existenz realisiert werden muss (Rn. 120).

Migrationspolitische Erwägungen, konkret die Verhinderung von Anreizen für Wanderungsbewegungen, können von vornherein ein Absenken des Leistungsstandards unter das einheitlich zu verstehende Existenzminimum nicht rechtfertigen, weil die Menschenwürde migrationspolitisch nicht zu relativieren ist (Rn. 121).

Kindern und Jugendlichen steht nicht der verfassungsrechtlich geforderte gesetzliche, d.h. bindende, Anspruch auf Leistungen für ihre spezifischen Bedarfe für Bildung und Teilhabe zu (Rn. 122).

\section{Verfassungsrechtliche Maßstäbe}

Ändert der Gesetzgeber nun entsprechend dem Auftrag des Bundesverfassungsgerichts das AsylbLG, so hält das Grundgesetz hierfür zwei zentrale grundrechtliche Vorgaben bereit, die miteinander verschränkt sind, gleichwohl aber den Blick auf zwei unterschiedliche Aspekte lenken. Zum einen stellt der unmittelbare verfassungsrechtliche Anspruch auf Gewährleistung eines menschenwürdigen Existenzminimums gemäß Art. 1 Abs. 1 GG i.V.m. dem Sozialstaatprinzip des Art. 20 Abs. 1 GG Anforderungen an den Gesetzgeber bei der Normierung des konkreten Leistungsanspruchs (unter 1.). Zum anderen fordert der allgemeine Gleichheitssatz des Art. 3 Abs. 1 GG sachliche Gründe, die die Bildung bestimmter - zueinander gleicher und gegenüber anderen unterschiedlicher - Personengruppen mit gleichen bzw. unterschiedlichen Leistungsansprüchen rechtfertigen kann (unter 2.).

\section{Gewährleistung eines menschenwürdigen Existenzminiums}

Die verfassungsrechtlichen Anforderungen, die aus der Gewährleistungspflicht für ein menschenwürdiges Existenzminimum folgen, hat das Bundesverfassungsgericht in seinem Urteil vom 18.7.2012 detailliert herausgearbeitet. Sie wurden bereits stichpunktartig zusammengestellt und sollen nun auf fünf Kernaussagen reduziert und diese erläutert werden. 


\section{a) Leistungsanspruch}

Die verfassungsrechtliche Gewährleistung verlangt eine gesetzgeberische Ausgestaltung, die dem einzelnen Berechtigten einen bindenden gesetzlichen Anspruch auf alle Leistungen gewährt, die Bestandteil seines menschenwürdigen Existenzminimums sind. Ermessensregelungen, durch die die für das Existenzminimum gebotenen Leistungen erbracht werden können, aber nicht erbracht werden müssen, genügen nicht.

\section{b) Leistungsbemessung}

Zentral ist die bereits im Urteil vom 9.2.2010 für die Regelsätze des SGB II aufgestellte Anforderung, dass die gesetzlich bestimmte Leistungshöhe folgerichtig in einem inhaltlich transparenten und sachgerechten Verfahren nach dem tatsächlichen und jeweils aktuellen Bedarf, also realitätsgerecht bemessen, begründbar sein muss. Dies schließt ein Abweichen von der Leistungsermittlung im Rahmen des SGB II und SGB XII nicht grundsätzlich aus. Der Gesetzgeber kann für einen besonderen Personenkreis den realitätsgerechten Bedarf auch mit einer anderen Methode bestimmen. ${ }^{41}$

Wählt er diesen Weg, so verlangt das Erfordernis eines folgerichtigen und sachgerechten Verfahrens, dass die gewählte Methode für den betroffenen Personenkreis sachgerecht ist. Je spezifischer der betroffene Personenkreis ist, desto inhaltlich anspruchsvoller gestaltet sich dieses Erfordernis. Denn die Bestimmung eines generell in Deutschland gebotenen Existenzminimums beruht grundsätzlich auf einer sachgerechten Tatsachengrundlage, wenn die generelle Lebenssituation in Deutschland Ausgangspunkt der Berechnungen ist. Aus diesem Grund ist die Einkommens- und Verbrauchsstichprobe für ein deutschlandweit festgelegtes Existenzminimum eine im Ansatz zulässige Grundlage. ${ }^{42}$ Soll hingegen für einen sehr engen, spezifischen Personenkreis die Leistungshöhe gesetzlich und damit generell bestimmt werden und soll hierfür eine auf diesen Personenkreis spezifisch zugeschnittene Bemessungsmethode verwendet werden, so muss diese Methode so beschaffen sein, dass sie zum einen sachgerechte Informationen über diesen spezifischen Personenkreis erfasst und zum anderen diese Informationen auch zuverlässig so generalisiert, dass sie als Grundlage für eine generelle, auf den Personenkreis zugeschnittene Leistungsbemessung geeignet sind. ${ }^{43}$ Es erscheint sehr zweifelhaft, dass es dem Gesetzgeber gelingen kann, eine empirische Basis für eine eigenständige Leistungsbemessung des vom AsylbLG betroffenen Personenkreises zu generieren, die diesen Anforderungen genügt. Bereits die Heterogenität der Aufenthaltsdauer, der Aufenthaltsgründe bzw. Ausreisehindernisse, sowie die unterschiedlichen Erwerbsberechtigungen der betroffenen Personen dürften es kaum möglich machen, dass für diese Gruppe eine einheitliche und von der allgemeinen Bevölkerung verschiedene Bedarfslage ermittelt werden kann.

Entscheidet sich der Gesetzgeber daher für den anderen Weg und bestimmt auch für einen besonderen Personenkreis den realitätsgerechten Bedarf nach der gleichen Methode, die er für andere Personen - im Rahmen des SGB II/SGB XII - auch verwandt

41 BVerfGE 116, 229, 239.

42 BVerfGE 125, 175, $234 \mathrm{ff}$.

43 Vgl. auch Fahlbusch, NDV 2011, 145. 
hat, so ist die Methodenwahl selbst nicht weiter rechtfertigungsbedürftig. Verfassungsrechtlicher Rechtfertigungsbedarf entsteht vielmehr zum einen dafür, dass ein bestimmter Personenkreis aus der generellen Regelung ausgegliedert wird. Dieser Aspekt wird im Folgenden unter dem Gesichtspunkt des Art. 3 Abs. 1 GG behandelt (s.u.). Zum anderen sind die Leistungsabweichungen gegenüber der allgemeinen Leistungsbemessung sachlich zu rechtfertigen. Konkret müssen aus den Spezifika der Lebenssituation des besonderen Personenkreises Ableitungen sachgerecht und nachvollziehbar möglich sein, die bestimmte abweichende Bedarfsbemessungen begründen. Diese Differenzierung der Leistungsbemessung ist Teil der von der Gewährleistungsverpflichtung geforderten folgerichtigen und sachgerechten Anspruchskonkretisierung durch den Gesetzgeber.

\section{c) Aufenthaltsstatus kein hinreichender Grund für abweichende Bedarfe}

Der Aufenthaltsstatus bestimmter Personengruppen kann für diese Spezifika der Lebenssituation ein Indiz sein. Aber allein der Aufenthaltsstatus genügt noch nicht, um bereits durch ihn einen abweichenden Bedarf zu begründen. Denn zwischen ausländerrechtlichem Status und typisierbarer Lebenssituation besteht kein hinreichend enger Zusammenhang. Die verschiedenen Aufenthaltstitel orientieren sich grundsätzlich am Aufenthaltszweck. Notwendigerweise tritt dabei ein Aufenthaltszweck als ausländerrechtlich ausschlaggebend in den Vordergrund, obwohl in der konkreten Lebenssituation nicht selten - womöglich sogar meistens - mehrere Aufenthaltszwecke nebeneinander stehen. So sind Menschen sowohl in einer Ausbildung als auch erwerbstätig. Oder sie nehmen ihren Aufenthalt in Deutschland, um mit ihren Familienangehörigen zusammen zu leben, sind dabei aber zugleich erwerbstätig oder in der Ausbildung. Menschen, die als Flüchtlinge nach Deutschland kommen oder Asyl suchen, haben womöglich zugleich auch Familienangehörige. Regelmäßig wollen (und sollen) sie auch erwerbstätig sein, etc. Dies bedeutet, dass der Aufenthaltstitel, der an einem Aufenthaltszweck anknüpft, oft nur Teilaspekte der Lebenssituation erfasst. Um aus Personen mit dem gleichen Aufenthaltstitel eine Gruppe zu bilden, die in spezifischer Weise abweichende Bedarfe gegenüber dem Bedarf, ,normaler“ Hilfebedürftiger hat, müssen deshalb weitere Merkmale dieser Personengruppe hinzutreten.

\section{d) Aufenthaltsperspektive als Grund für abweichende Bedarfe}

Ein solches - zum gemeinsamen Aufenthaltstitel hinzutretendes - Merkmal kann die Aufenthaltsperspektive sein. Die Besonderheit der leistungsberechtigten Personengruppe des AsylbLG gegenüber anderen Hilfebedürftigen wäre dann in einer fehlenden längerfristigen Aufenthaltsperspektive zu sehen. An dieser Stelle kommt es nicht darauf an, ob die Personengruppen, die dem AsylbLG zugewiesen werden, tatsächlich Menschen ohne längerfristige Aufenthaltsperspektive sind. Dieser Frage ist später bei der Rechtfertigung der Differenzierung nachzugehen (s.u.).

Wenn man aber unterstellt, dass es eine Personengruppe gibt, die keine längerfristige Aufenthaltsperspektive hat, so ist die Frage zu beantworten, wie sich aus dem Fehlen dieser Aufenthaltsperspektive Abweichungen hinsichtlich des menschenwürdigen Exis- 
tenzminimums ergeben. Eine solche plausible Abweichung wäre etwa der Verzicht auf Leistungen für den Hausrat bei hilfebedürftigen Touristen, die keine Wohnung in Deutschland nehmen, sondern in ihren Herkunftsstaat zurückkehren. Diese Konstellation ist freilich für die bisher nach dem AsylbLG Berechtigten nicht relevant, da sie keine Touristen sind. Gleichwohl lässt sich vorstellen, dass allgemein Personen ohne längerfristige Aufenthaltsperspektive gegenüber in Deutschland lebenden Menschen abweichende Bedarfe haben. Will der Gesetzgeber solche Abweichungen vorsehen, so müssen sie mit gerade dem Fehlen einer längerfristigen Aufenthaltsperspektive erklärbar sein.

Das Touristenbeispiel macht noch einen zweiten Aspekt deutlich. Je kürzer die Aufenthaltsperspektive ist, desto plausibler ist der Verzicht auf bestimmte Bedarfe - etwa (weiterer) Kleidung oder auch Teilhabe an Bildung, wenn ein Aufenthalt nur wenige Tage oder Stunden dauert. Umgekehrt sind Abweichungen vom Normalfall umso schwerer erklärbar, je länger der Aufenthalt ist. Bei Wohnsitznahme bzw. ab einer Aufenthaltsperspektive, die typischerweise mit einer Wohnsitznahme einhergeht, sind Abweichungen gegenüber anderen Hilfebedürftigen kaum noch plausibel.

\section{e) Vermeidung von Migrationsanreizen kein Kriterium zur Leistungsbemessung}

Das zweite Motiv, das der bisherigen Regelung bisweilen beigemessen wurde (s.o.), ist das der Vermeidung von Migrationsanreizen. Wiederum sei an dieser Stelle zunächst unterstellt, die Personengruppe, die von der spezifischen Leistungsbemessung betroffen ist, sei die gleiche, bei der der Anreiz zur Migration nach und zum Bleiben in Deutschland vermieden werden soll. In diesem Fall bleibt für die Beachtung der Gewährleistungspflicht für ein menschenwürdiges Existenzminimum die Frage zu beantworten, ob der Leistungsbedarf dieser Personen dadurch sinkt, dass ihnen keine Migrationsanreize gesetzt werden: Hat eine Person, für die kein Migrationsanreiz bestehen sollte, die aber gleichwohl nach Deutschland gekommen ist, einen niedrigeren Bedarf? Entsprechendes gilt für das Vermeiden von Anreizen zum Bleiben: Ist das menschenwürdige Existenzminimum dadurch niedriger, dass kein Anreiz bestehen soll, zu bleiben? Das Bundesverfassungsgericht hat die Frage eindeutig verneint. In der Tat ist nicht plausibel, warum der Bedarf der betroffenen Personen niedriger ist. Damit lässt sich eine abgesenkte Leistungshöhe nicht mit dem Ziel der Vermeidung von Migrationsanreizen begründen.

Das gesetzgeberische Ziel, keine Migrationsanreize zu setzen, ist dabei für sich genommen nicht verfassungsrechtlich problematisch. Fraglich ist vielmehr, welche staatlichen Mittel dieses Ziel rechtfertigen kann. Eingriffe in bestimmte Grundrechte mögen sich auf diese Weise rechtfertigen lassen. Die gesetzliche Konkretisierung des grundrechtlichen Anspruchs auf Gewährleistung des menschenwürdigen Existenzminimums darf dieses Ziel aber nicht beeinflussen. Denn der tatsächliche Bedarf von Menschen, die in Deutschland leben, unterscheidet sich nicht danach, ob sie mit oder ohne vom deutschen Recht gesetzten Migrationsanreizen nach Deutschland gekommen sind. 


\section{f) Zusammenfassung}

Es zeigt sich, dass Abweichungen von der generellen Leistungsbestimmung, die der Gesetzgeber für das SGB II/SGB XII getroffen hat, zwar verfassungsrechtlich nicht ausgeschlossen sind. Will aber der Gesetzgeber für bestimmte Gruppen von Ausländern abweichende Bedarfe normieren, so begegnet er gewichtigen Hürden. Der Aufenthaltsstatus ist kein hinreichendes Kriterium, um allein darauf gestützt einen vom Normalfall abweichenden Bedarf verfassungsrechtlich hinreichend plausibel zu bestimmen. Stellt man daher (auch) auf die Aufenthaltsperspektive ab, so lässt sich damit nur ein relativ kleiner Kreis von Personen mit Kurzaufenthaltsperspektive erfassen. Unzulässig ist es jedenfalls, die Bedarfsermittlung davon leiten zu lassen, dass Migrationsanreize vermieden werden sollen.

\section{Sachliche Differenzierungs- und Gleichstellungsgründe}

Über die bisher erörterte Frage hinaus, wie sich abweichende Bedarfe einer bestimmten Personengruppe sachlich begründen lassen, muss ein AsylbLG, dass diesen abweichenden Bedarf für bestimmte Personengruppen vorgibt, auch insoweit verfassungsrechtlichen Anforderungen genügen, dass diese Personengruppen sachlich vertretbar bestimmt wurden. Maßstab hierfür ist Art. 3 Abs. 1 GG.

Der allgemeine Gleichheitssatz gebietet dem Gesetzgeber, wesentlich Gleiches gleich und wesentlich Ungleiches ungleich zu behandeln. ${ }^{44}$ Dies bedeutet im hier interessierenden Zusammenhang, dass die Gruppen von Normadressaten, die nicht dem SGB II/ SGB XII, sondern dem AsylbLG mit dem dort abweichend festgesetzten Bedarf zugewiesen wird, gegenüber den SGB II/SGB XII-Leistungsbeziehern Unterschiede von solcher Art und solchem Gewicht aufweisen müssen, dass sie diese unterschiedliche Behandlung rechtfertigen. Umgekehrt müssen die im AsylbLG zusammengefassten Personengruppen in Bezug auf die Höhe ihres menschenwürdigen Existenzminimums untereinander im Wesentlichen gleich sein. Denn andernfalls läge in der Gleichbehandlung von wesentlich Ungleichem ebenfalls ein Verstoß gegen den allgemeinen Gleichheitssatz.

\section{a) Entscheidung des Bundesverfassungsgerichts vom 10.7.2012}

An einer aktuellen Entscheidung des Bundesverfassungsgerichts zur Frage, ob bestimmte Ausländergruppen von Erziehungs- bzw. Elterngeld ausgeschlossen werden dürfen, ${ }^{45}$ ist deutlich erkennbar, wie dieser gleichheitsrechtliche Maßstab anzuwenden ist.

Ausgangspunkt sind die Ziele, die der Gesetzgeber mit der von ihm getroffenen Differenzierung verfolgt. In der Entscheidung zum Erziehungs- und Elterngeld orientiert sich das Bundesverfassungsgericht an den gesetzgeberischen Ziel, Eltern die eigene Betreuung ihres Kindes durch Verzicht auf eine Erwerbstätigkeit oder durch deren Ein-

44 St. Rspr., vgl. nur BVerfG, Beschluss vom 10.7.2012 - 1 BvL 2/10 u.a., Rn. 37.

45 BVerfG, Beschluss vom 10.07.2012 - 1 BvL 2/10u.a. 
schränkung zu ermöglichen (Rn. 41). Ebenso ist die Intention des Gesetzgebers zulässig, Erziehungs- oder Elterngeld nur Personen zu gewähren, die sich voraussichtlich auf Dauer in Deutschland aufhalten werden (Rn. 42).

An diesem Ziel sind sodann die Kriterien zu messen, mit denen der Gesetzgeber die betroffene Personengruppe erfasst. Im Fall des Erziehungs- und Elterngeldes ist etwa die Voraussetzung, dass Ausländer überhaupt erwerbsfähig sind, ein sachlich zulässiges Kriterium. Denn bei Personen, die gar nicht erwerbstätig sein dürfen und daher nicht erwerbsfähig sind, kann das Erziehungs- bzw. Elterngeld die vom Gesetzgeber intendierte Chance, auf Erwerbstätigkeit zu verzichten, nicht bieten (Rn. 41). Die Voraussetzung, dass die Personen tatsächlich erwerbstätig (gewesen) sein muss, ist hingegen nicht geeignet, um den Personenkreis zu identifizieren, der den Intentionen des Gesetzgebers bei der Leistungsgestaltung entspricht. Denn daraus, dass Personen bis zur Beantragung des Erziehungs- oder Elterngeldes nicht erwerbstätig waren, lässt sich keine Prognose ableiten, dass ihr Aufenthalt nicht auf Dauer angelegt ist (Rn. 46 ff.).

Gleiches gilt auch für den Aufenthaltstitel. Allein der Aufenthaltstitel ,ist kein hinreichendes Indiz für das Fehlen einer dauerhaften Aufenthaltsperspektive“ (Rn. 44). Dies gilt, wie das Bundesverfassungsgericht ausführt, namentlich für die Aufenthaltstitel des 5. Abschnitts des Aufenthaltsgesetzes und damit für die auch im Rahmen des AsylbLG relevanten $\S 25$ Abs. 4 bis 5 AufenthG. Die aufenthaltsrechtliche Konstruktion dieser Aufenthaltstitel zeigt bereits, dass sich aus dem Titel selbst keine verlässliche Aufenthaltsprognose ableiten lässt. Denn gemäß § 26 AufenthG sind diese Titel stets befristet, teilweise auch auf maximal sechs Monate. Aber bei Fortbestehen der humanitären, politischen oder völkerrechtlichen Aufenthaltsgründe kommt bei fast allen Titeln eine Verlängerung in Betracht, die uneingeschränkt wiederholbar ist. Zudem sieht $\S 26$ Abs. 4 AufenthG nach sieben Jahren die Möglichkeit einer Niederlassungserlaubnis vor. Die Aufenthaltstitel aus humanitären, politischen oder völkerrechtlichen Gründen sind daher nach der Konzeption des AufenthG einer Verfestigung gerade zugänglich (Rn. 45). Je länger im Einzelfall der Aufenthalt in Deutschland ist, desto höher ist dadurch die Wahrscheinlichkeit, dass die Verfestigung später eintritt.

\section{b) Geeignete Differenzierungsgründe für das AsylbLG}

Für die verfassungsrechtliche Bewertung der Zuordnung von Personengruppen zum AsylbLG lassen sich diese Ausführungen des Bundesverfassungsgerichts übertragen. Ausgangspunkt ist dabei die Konzeption, die der Gesetzgeber durch die abweichende Bestimmung des Existenzminimums verfolgt. Je nachdem, wie er das vom Regelbedarf des SGB II/XII abweichende menschenwürdige Existenzminimum des AsylbLG ermittelt (s.o.), müssen die Kriterien, mit denen die Leistungsberechtigten bestimmt werden, beschaffen sein, damit die „richtigen“ Personengruppen erfasst werden, die tatsächlich dem gesetzgeberischen Leitbild des Leistungsberechtigten entsprechen.

\section{(1) Existenzminimum bei Kurzaufenthalt - Personenkreis mit Kurzaufenthalt}

Falls der Gesetzgeber das menschenwürdige Existenzminimum der Leistungsbezieher des AsylbLG anhand des Leitbildes ermittelt, dass die Leistungsbezieher sich nur kurz 
- etwa typischerweise drei Monate - in Deutschland aufhalten, so verlangt der allgemeine Gleichheitssatz, dass die Personengruppen, die dem AsylbLG zugewiesen sind, sich tatsächlich typischerweise höchstens drei Monate in Deutschland aufhalten. Das Bundesverfassungsgericht verlangt wörtlich: „Lassen sich tatsächlich spezifische Minderbedarfe bei einem nur kurzfristigen, nicht auf Dauer angelegten Aufenthalt feststellen, und will der Gesetzgeber die existenznotwendigen Leistungen für eine Personengruppe deshalb gesondert bestimmen, muss er sicherstellen, dass die gesetzliche Umschreibung dieser Gruppe hinreichend zuverlässig tatsächlich nur diejenigen erfasst, die sich regelmäßig nur kurzfristig in Deutschland aufhalten. ${ }^{\text {"46 }}$ Entsprechendes gilt, wenn der Gesetzgeber der Bedarfsberechnung einen zu erwartenden Aufenthalt von anderer Länge, etwa sechs oder zwölf Monaten, zugrunde legt.

Diesen gleichheitsrechtlichen Anforderungen kann der Gesetzgeber relativ einfach dadurch genügen, dass er bei der Zuweisung der Personengruppen zum AsylbLG folgende Aspekte berücksichtigt: Die Dauer des bisherigen Aufenthalts in Deutschland gemeinsam mit der Befristung des konkreten Aufenthaltstitels gibt einen plausiblen Anhaltspunkt dafür, dass eine Person sich so kurz in Deutschland aufhalten wird, wie es dem Leitbild der Personengruppe entspricht, die der Gesetzgeber bei der Bedarfsermittlung des AsylbLG zugrunde gelegt hat.

Seine Grenze hat diese Anknüpfung an die Befristung des Aufenthaltstitels dort, wo das AufenthG zwingende Befristungen eines Aufenthaltstitels vorgibt. Denn in diesen Fällen beruht die konkrete Aufenthaltsfrist nicht auf der Aufenthaltsprognose im Einzelfall, sondern auf der aufenthaltsrechtlichen Grundentscheidung, bestimmte Aufenthaltstitel nur für bestimmte Dauer zu gewähren und dadurch das Fortbestehen der Aufenthaltsvoraussetzungen immer wieder neu zu prüfen. Namentlich gilt dies für die humanitären Aufenthaltstitel des $\S 25$ Abs. 4 bis 5 AufenthG. Gemäß $\S 26$ Abs. 1 AufenthG sind diese Aufenthaltstitel entweder bindend (im Fall des $\S 25$ Abs. $4 \mathrm{a}$ und 4 b AufenthG) oder längstens (im Fall des $\S 25$ Abs. 4 S. 1 und Abs. 5 AufenthG) für sechs Monate zu gewähren. Dies bedeutet, dass auch Personen, bei denen aufgrund der tatsächlichen Begebenheiten eine längere Aufenthaltsperspektive besteht, nur ein kurz befristeter Aufenthaltstitel erteilt wird. Das Bundesverfassungsgericht hat in seiner Entscheidung zum Erziehungs- und Elterngeld deutlich gemacht, dass sich gerade bei Aufenthaltstiteln aus humanitären, politischen oder völkerrechtlichen Gründen allein aus dem Aufenthaltstitel noch keine solche Prognose für die Aufenthaltsdauer ableiten lässt, dass Rechtsfolgen auf der Annahme eines nicht dauerhaften Aufenthalts beruhen können. ${ }^{47}$ Dies gilt noch mehr, wenn die Prognose nicht nur darauf gerichtet ist, ob der Aufenthalt letztlich auf Dauer sein wird, sondern darauf zielt, dass der Aufenthalt besonders kurz ist. Abstrakt formuliert büßt die Dauer des Aufenthaltstitels ihre Indizwirkung für die sozialrechtliche Zuordnung zu einem abweichenden Existenzminimum dadurch ein, dass das Aufenthaltsrecht aufgrund seiner eigenen Binnenrationalität die Aufenthaltsdauer gesetzlich vorgibt.

Es bedarf keiner weiteren Erörterung, dass die Anknüpfung an den tatsächlichen Aufenthaltsbeginn nicht durch andere Parameter, etwa des Beginns des Leistungsbezugs ersetzt werden kann. Denn nur bei Anknüpfung an den Aufenthaltsbeginn ist sicherge-

46 BVerfG, Urteil vom 18.7.2012 - 1 BvL 10/10 und 1 BvL 2/11, Rn. 10.

47 BVerfG, Beschluss vom 10.7.2012 - 1 BvL 2/10 u.a., Rn. 43 ff.; s. bereits oben. 
stellt, dass die erfassten Personen sich voraussichtlich so lange in Deutschland aufhalten werden, wie der Gesetzgeber bei der Bedarfsbestimmung zugrunde gelegt hat.

(2) Existenzminimum während Asylverfahren - Personenkreis mit Gestattung gemäß $\S 55$ AsylVerfG

Entsprechendes gilt, wenn der Gesetzgeber etwa das menschenwürdige Existenzminimum des AsylbLG auf der Grundlage ermittelt, welche Bedarfe Personen während eines laufenden Asylverfahrens tatsächlich haben.

In diesem Fall ist die Anknüpfung an die Aufenthaltsgestattung nach dem $§ 55$ AsylVerfG ein grundsätzlich taugliches Mittel, um den Personenkreis zu ermitteln, der dem Leitbild des Gesetzgebers entspricht.

Aber auch hier hat der Gesetzgeber zu berücksichtigen, dass die ausländerrechtliche Zuordnung unter Umständen auch Personen betrifft, bei denen die Annahmen, die der Gesetzgeber bei der Leistungsbestimmung zugrunde gelegt hat, nicht zutreffen. Namentlich bei Asylanträgen, die nicht unmittelbar bei oder in zeitlichem Zusammenhang mit der Einreise gestellt werden, ist fraglich, ob ein hinreichender sachlicher Grund besteht, auch bei diesen Personen von dem asylbewerberspezifischen Existenzminimum auszugehen. Daher wäre bei einer Leistungsbemessung, die sich am besonderen Bedarf der (Erst-)Asylantragsteller unmittelbar nach ihrer Einreise orientiert, $\S 1$ Abs. 1 Nr. 7 AsylbLG in seiner heutigen Fassung aus Gleichheitsgesichtspunkten nicht mehr verfassungsrechtlich haltbar, weil er Personen unabhängig vom Voraufenthalt erfasst.

Sollte der Gesetzgeber andere Personengruppen identifizieren, die er seiner spezifischen Bedarfsermittlung zugrundlegt, sind die angestellten Erwägungen entsprechend zu übertragen.

\section{Leistungsänderungen des AsylbLG}

Seit dem Urteil des Bundesverfassungsgerichts wird diskutiert, wie ein verfassungskonformes AsylbLG auszusehen hat. Insbesondere von Organisationen, die die Interessen von Flüchtlingen vertreten, wird die Abschaffung des AsylbLG gefordert. ${ }^{48}$ Auch einige Landesregierungen ${ }^{49}$ sowie die Bundestagsfraktion Bündnis 90/Die Grünen ${ }^{50}$ haben sich dieser Forderung angeschlossen. Auf der anderen Seite des politischen Spektrums schlägt Bundesinnenminister Friedrich vor, für Personen aus bestimmten EUAnrainerstaaten, konkret Serbien und Mazedonien, die Barleistungen des AsylbLG zu

48 Etwa Pro Asyl e.V.: http://www.proasyl.de/de/presse/detail/news/urteil_des_bundesverfass ungsgerichts_zum_asylbewerberleistungsgesetz/ (8.12.2012); bereits 2011 sprachen sich bei einer Sachverständigenanhörung des Bundestages der Bevollmächtigte der Evangelischen Kirche, der Flüchtlingsrat Berlin e.V., die Bundesarbeitsgemeinschaft der Freien Wohlfahrtspflege; der Deutsche Verein für öffentliche und private Fürsorge und der Deutsche Caritasverband e.V. für eine Abschaffung des AsylbLG aus, BT-Drs. 17/10198.

49 Antrag der Bundesländer Rheinland-Pfalz, Brandenburg und Schleswig-Holstein, BR-Drs. $576 / 12$.

50 BT-Drs. 17/11707; schon vor dem Urteil BT-Drs. 17/1428; auch Die Linke, BT-Drs. 17/4424. 
streichen oder zumindest stark zu reduzieren. ${ }^{51}$ In der Diskussion finden sich außerdem unterschiedliche Auffassungen dazu, ob und inwieweit Sach- statt Geldleistungen möglich bleiben. ${ }^{52}$ Weitgehend unstreitig scheint zu sein, dass das Teilhabe- und Bildungspaket auch Beziehern von Asylbewerberleistungen zustehen muss. Im Folgenden sollen daher einzelne Änderungsoptionen auf ihre Verfassungsmäßigkeit bzw. auf ihre verfassungsmäßige Ausgestaltung hin beleuchtet werden.

\section{Sachleistungen}

\section{a) Allgemeine Erwägungen für Sachleistungen zur Existenzsicherung}

Eine solche alternative Leistungserbringung und damit verknüpft auch Leistungsbemessung liegt grundsätzlich im Gestaltungsspielraum des Gesetzgebers und ist daher verfassungsrechtlich nicht ausgeschlossen.

Sie muss aber für sich genommen sachgerecht sein. Dies verlangt zum einen die grundrechtliche Gewährleistungspflicht für die Sicherstellung des menschenwürdigen Existenzminimums (Art. 1 Abs. 1 GG i.V.m. Sozialstaatsprinzip). Zugleich bildet die leistungsbezogene Sachgerechtigkeit den sachlichen Grund für die Differenzierung, welche zwischen den Personengruppen vorgenommen wird, die diesem Leistungssystem zugewiesen werden, und den anderen Personengruppen, deren Existenzminimum in den Regelleistungssystemen des SGB II/SGB XII gesichert ist (Art. 3 Abs. 1 GG).

In der aktuellen politischen Diskussion werden Sachleistungen generell als entwürdigend abgelehnt. ${ }^{53}$ In der rechtswissenschaftlichen Debatte hat Rothkegel jüngst in Zweifel gezogen, dass ein Sachleistungssystem diesen Anforderungen genügen kann. ${ }^{54}$ Es sei zweifelhaft, ob die Gewährung von Sachleistungen den Anforderungen an eine verfassungskonforme Leistungsbemessung genügen kann. Auch sei bei dieser Form der Leistungserbringung die Wahrung der Autonomie der Leistungsberechtigten gefährdet und eine diskriminierende Wirkung möglich. Dem kann in dieser Pauschalität nicht gefolgt werden. Rothkegel misst im Ergebnis die Gewährung von Sachleistungen an den Maßstäben, die für die pauschalierte Geldleistung des SGB II entwickelt wurden. Sachleistungen sind jedoch stets konkrete Güter und gerade nicht pauschal, dadurch stellen sich Fragen der Leistungsbemessung anders. Verfassungsrechtlich ausschlaggebend ist nicht, ob sie auf einer ebensolchen Datenbasis beruht, wie die Regelleistungsbemessung. Entscheidend ist, dass die gesetzliche Leistungsbestimmung sachgerecht ist und solche Maßstäbe bietet, dass die (Sach-)Leistungserbringung durch die Verwaltungsbehörden den grundrechtlichen Anforderungen entspricht. Das betrifft insbesondere die Qualität der Sachleistungen, etwa die Frage der Neuwertigkeit. Das be-

51 http://www.bild.de/politik/inland/hans-peter-friedrich/innenminister-klagt-ueber-fluechtling s-ansturm-auf-deutschland-26683892.bild.html (abgerufen am 7.12.12).

52 S. etwa Entschließungsantrag der SPD-Bundestagsfraktion, BT-Drs. 17/11674, Nr. 6 und 7.

53 Schon 2010 Pro Asyl e.V.: http://www.proasyl.de/en/topics/rechtspolitik/stellungnahmen / 2010/sachleistungsprinzip/ (abgerufen 7.12.2012); aktuell etwa Gemeinsame Presseerklärung des Flüchtlingsrates Schleswig-Holstein e.V. und Pro Asyl e.V. vom 30.11.2012: http:// www.frsh.de/fileadmin/pdf/presseerklaerungen/2012/pe_frsh-proasyl_30.11.2012.pdf (abgerufen 8.12.2012).

54 Rothkegel, ZAR 2011, 90. 
deutet auch, dass die Leistungserbringung einer gerichtlichen Prüfung zugänglich sein muss. Dies sind jedoch Fragen der gesetzlichen Ausgestaltung und führen nicht schon im Ansatz zur Verfassungswidrigkeit von Sachleistungsgewährung für das menschenwürdige Existenzminimum.

\section{b) In Erstaufnahmeeinrichtungen Sachleistungen}

Die Beibehaltung der bisherigen Leistungserbringung in Form von Sachleistungen - mit Ausnahme eines Barbetrags für die Bedürfnisse des täglichen Lebens - während der Unterbringung in Erstaufnahmeeinrichtungen mit einer zeitlichen Begrenzung auf höchstens drei Monate des Aufenthalts ist verfassungsrechtlich nicht zu beanstanden, wenn die Abweichung von der Leistungserbringung ,im Normalfall“", also des SGB II/ SGB XII sachlich begründbar ist. Dies ist grundsätzlich bei einer Unterbringung in einer öffentlichen Einrichtung der Fall. Dass sich hierbei die erbrachten Leistungen nach den Gegebenheiten der Erstaufnahmeeinrichtung richten und nicht vom Gesetz bis ins Detail vorgegeben werden, ist ebenfalls verfassungsrechtlich unbedenklich. Das Sozialrecht kennt in verschiedenen Bereichen die Erbringung von Sachleistungen, deren konkrete Gestalt erst im Einzelfall bestimmt wird. Verfassungsrechtlich relevant ist in diesem Zusammenhang nur, dass auf diese Leistungen gleichwohl ein gesetzlicher Anspruch besteht, es sich also um gebundene Verwaltungsentscheidungen handelt und nicht um Ermessensleistungen.

\section{c) Grundsätzlicher Vorrang von Geldleistungen mit einer Öffnungsklausel für die Bundesländer, um die Leistungserbringung nach dem Sachleistungsprinzip vorzunehmen}

Um unterschiedlichen Interessen und Vorstellungen der Bundesländer Rechnung zu tragen, wäre es vorstellbar, den Bundesländern einen Gestaltungsspielraum bei der Leistungsform einzuräumen. Da die Verwaltungskompetenz nach den Grundsätzen des deutschen Föderalismus grundsätzlich bei den Ländern liegt (Art. 83 ff. GG), sind solche Gestaltungsspielräume staatsorganisationsrechtlich nicht zu beanstanden. Auch die grundrechtlichen Vorgaben führen zu keiner anderen föderalen Zuordnung. Vielmehr erheben die Grundrechte des Grundgesetzes gegenüber der ausführenden Verwaltung der Bundesländer, aber auch gegenüber landesgesetzgeberischer Ausgestaltung, den gleichen Geltungsanspruch. Das bedeutet für die aufgeworfene Frage:

Der Bundesgesetzgeber darf alternativ Sach- oder Geldleistungsgewährung vorsehen, so wie er sich auch für Sach- oder für Geldleistungen entscheiden könnte. Grundrechtlich ist dabei stets „,nur“ ausschlaggebend, dass das gewählte Leistungskonzept und die darin enthaltene Leistungsbemessung methodisch den Anforderungen des Bundesverfassungsgerichts entsprechen. Räumt er dem Landesgesetzgeber oder der Landesverwaltung insoweit Gestaltungsspielraum ein, so stellen sich die gleichen Anforderungen auf dieser Ebene. Der Landesgesetzgeber oder der Landesverordnungsgeber müssen ihre Ausgestaltungsregelung auf einer belastbaren Tatsachengrundlage in einem transparenten und nachvollziehbaren Verfahren darlegen können. 
d) Sachleistungsprinzip (als Ausnahme vom Geldleistungsgrundsatz) für eine angemessene Erstausstattung

\section{(1) Erstausstattung als Sachleistung}

Diskutiert wird darüber hinaus, eine Erstausstattung in Form von Sachleistungen zu gewähren und dafür entsprechend die Bedarfspositionen der Geldleistung für einen bestimmten Zeitraum zu kürzen (hierzu sogleich). Konkret besteht die Vorstellung, die Bedarfe an Hausrat im Rahmen einer Erstausstattung zu gewähren. ${ }^{55}$

Dies wäre dann eine Entscheidung für ein Leistungssystem, das mit einer anderen Bedarfsbemessung verknüpft ist als das Regelleistungssystem des SGB II/SGB XII. Sie beruht auf der Idee, neu nach Deutschland eingereisten Asylbewerberinnen und Asylbewerbern sowohl in der Erstaufnahmeeinrichtung als auch im Anschluss an diese Zeit eine Erstausstattung zur „Einrichtung“ eines menschenwürdigen Daseins in der Bundesrepublik Deutschland zu gewähren. Leitbild dieses Konzeptes sind hilfebedürftige Personen, die mit wenig Habe, jedenfalls ohne Hausstand und regelmäßig auch mit für die deutschen Rahmenbedingungen unzureichender Kleidung, nach Deutschland einreisen. Wenn dies der tatsächlichen Situation der Personengruppen entspricht, die dem AsylbLG zugewiesen werden sollen - etwa wenn dies für Asylbewerberinnen und Asylbewerber zutrifft - so ist dieses Leistungskonzept sachlich nachvollziehbar. Damit genügt es dem Grunde nach den verfassungsrechtlichen Anforderungen.

\section{(2) Dauer des Sachleistungsbezugs}

Fraglich ist jedoch, für welchen Zeitraum dieses Sachleistungskonzept sachlich begründbar ist. Würde wegen der Sachleistung der Geldleistungsbetrag während der gesamten Bezugsdauer des AsylbLG abgesenkt, dann müsste der sachliche Grund hierfür darin liegen, dass für die gewährten Sachleistungen eine Haltbarkeitsdauer über diesen Zeitraum angenommen werden kann. Denn anschließend sind im Rahmen der sog. Analogleistungen Beträge entsprechend dem Regelbedarf nach dem SGB II/SGB XII vorgesehen. Dort werden die regelmäßig anfallenden Kosten für die Bedarfspositionen auch etwa des Hausrates in monatlichen Anteilsbeträgen berechnet. Der Gesetzgeber geht dabei davon aus, dass Leistungsbezieher grundsätzlich über einen eingerichteten Haushalt verfügen, so dass keine neue Gesamtausstattung notwendig ist, sondern ein kontinuierliches Ersetzen einzelner Güter erfolgen kann. Wenn der Gesetzgeber für Leistungsberechtigte nach dem AsylbLG dieses System erst nach dem Vorbezugszeitraum vorsehen sollte, dann läge dem die Annahme zugrunde, dass zu diesem Zeitpunkt die als Sachleistung gewährte Erstausstattung regelmäßig den Zustand hat, den entsprechende Güter bei Leistungsbeziehern nach dem SGB II/SGB XII auch haben. Die Leistungsberechtigten könnten dann damit beginnen, für spätere Neubeschaffungen Geldbeträge anzusparen.

Ob diese Annahme sachlich gerechtfertigt ist, hängt davon ab, welche Leistungen der Gesetzgeber im Rahmen der Erstausstattung vorsieht und wie lang dieser Zeitraum des Sachleistungsbezugs sein soll. Dies lässt sich an zwei Beispielen veranschaulichen:

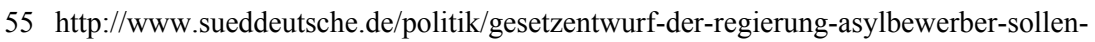
mehr-geld-bekommen-1.1537641 (abgerufen 8.12.2012). 
Wenn etwa eine neue Waschmaschine als Sachleistung gewährt wird, ist die gesetzliche Annahme nachvollziehbar, dass typischerweise in den ersten zwei Jahren - also der gesetzlichen Gewährleistungsfrist - und auch noch darüber hinaus kein Ersatz beschafft werden muss. Die Dauer des Sachleistungsbezugs äußert sich in diesem Fall dann darin, dass nur im atypischen Ausnahmefall, wenn die Waschmaschine doch unreparierbar defekt ist, eine neue Waschmaschine ebenfalls als Sachleistung gewährt wird. Bei dieser Konzeption ist eine Sachleistung über die Dauer von zwei Jahren und damit korrespondierend die Absenkung des Grundleistungsbetrags (s.u. 2.b.) sachlich nachvollziehbar und verfassungsrechtlich nicht zu beanstanden.

Etwas anderes gilt jedoch, wenn etwa Kinderschuhe als Sachleistung gewährt werden sollten. Typischerweise ist die Lebensdauer von Kinderschuhen weniger als ein Jahr. Die Gewährung einer Erstausstattung in Form von Sachleistungen deckt daher auch beim regelhaft zu erwartenden Verlauf nicht den Bedarf an Kinderschuhen der gesamten Vorbezugszeit, wenn diese weiterhin vier Jahre betragen würde. In diesem Fall würde die Dauer des Sachleistungsbezugs daher typischerweise auch die Ersetzung der Kinderschuhe bedeuten. Damit dieser wiederholte Sachleistungsbezug als Abweichung vom Grundkonzept gerechtfertigt ist, braucht er einen sachlichen Grund. Dieser war jedoch nur bei der Erstausstattung gegeben (s.o. unter (1)). Für Kinderschuhe erscheint daher ein Sachleistungsbezug nur für etwa 6 Monate gerechtfertigt.

Die Überlegung, die den Beispielen zugrunde liegt, lässt sich verallgemeinern: Die Dauer des Sachleistungsbezugs - und damit die Absenkung des Grundleistungsbetrags um die entsprechenden Bedarfspositionen - ist nur so lange gerechtfertigt, wie typischerweise ein ,durchschnittlicher Abnutzungszustand“ der entsprechenden Güter angenommen werden kann. Hierbei darf der Gesetzgeber typisieren, muss sich aber insgesamt an nachvollziehbaren Tatsachengrundlagen orientieren.

\section{Bedarfsbestimmung der Geldleistungen}

\section{a) Bedarfsermittlung auf der Grundlage der EVS 2008}

Wenn der Gesetzgeber für die Bedarfsbestimmung des AsylbLG auf der Grundlage der Einkommens- und Verbrauchs-Stichprobe (EVS) 2008 vornimmt, so folgt er dem oben erläuterten Weg, der keine eigenständige Methode für das AsylbLG vorsieht. Die Methode selbst ist daher verfassungsrechtlich nicht zu beanstanden. Rechtfertigungsbedürftig sind hingegen Abweichungen, die der Gesetzgeber von der Bemessung für das SGB II/SGB XII macht.

\section{b) Absenkung wegen Sachleistungen der Erstausstattung}

Knüpft man die Absenkung der Grundleistung daran an, dass für die entsprechende Bedarfsposition im Rahmen der Erstausstattung Sachleistungen gewährt wurden, und unterstellt man hierfür eine verfassungskonforme Dauer der Absenkung (s.u.), so birgt diese Isolierung von Sachleistungspositionen noch ein weiteres Problem:

Die Gewährung von Regelbedarfen zur Sicherung des menschenwürdigen Existenzminimums beruht auf dem Konzept, Hilfebedürftige mit einem Geldbetrag auszustatten, 
der ihnen Autonomie und Gestaltungsfreiheit bei der Deckung ihrer Bedarfe ermöglicht. Aus diesem Grund können für alle Bedarfspositionen statistische monatliche Durchschnittswerte angesetzt werden. Die pauschalierte Geldleistung beruht auf der Annahme, dass die Leistungsberechtigten teilweise durch Ansparen von Beträgen und teilweise durch „Umschichtung“ der rechnungsmäßigen Beträge auf andere Bedarfspositionen die Regelleistung so verwenden werden, dass ihr Existenzbedarf gedeckt ist. ${ }^{56}$

Je geringer der Pauschalbetrag ist, desto weniger Spielraum besteht für diese von Gesetzgeber gewollten und vorausgesetzten Umschichtungen. Dies gilt umso mehr, je größer der Anteil der Bedarfspositionen innerhalb des Pauschalbetrags ist, die der Befriedigung kurzfristiger Bedürfnisse dienen. Auf diese Weise gerät das Leistungsmodell der pauschalierten Grundleistung an seine Grenzen, wenn aus dem Gesamtbedarf zu viele Einzelbedarfe herausgelöst werden, die als Sachleistungen erbracht werden. Ab wann dies der Fall ist, lässt sich erst anhand konkreter Modellberechnungen entscheiden, die diesen Aspekt der „Tauglichkeit“ des verbleibenden Geldbetrages berücksichtigen.

\section{c) Absenkungen für die Bereiche Freizeit, Unterhaltung und Kultur}

In der Debatte ist auch zu hören, dass die Leistungen, die im Regelsatz nach SGB II/ SGB XII für die Bereiche Freizeit, Unterhalt und Kultur vorgesehen sind, bei Berechtigten nach dem AsylbLG niedriger festgesetzt werden könnten.

Eine verfassungskonforme Begründung hierfür müsste etwa dem Gedanken folgen, der mit einem fiktiven Beispiel veranschaulicht werden soll: Im Regelbedarf des SGB II/SGB XII sei von zwei Theaterbesuchen pro Jahr auszugehen. Da das AsylbLG in diesem Beispiel nur Personen in den ersten sechs Monaten ihres Aufenthaltes in Deutschland erfasst, werden Kosten für Theaterbesuche nicht berücksichtigt. Denn es wird auch Leistungsberechtigten nach dem SGB II/SGB XII zugemutet, durchschnittlich sechs Monate auf Theaterbesuche zu verzichten. Es zeigt sich also, dass die Streichung einer bestimmten Position auch in diesen Bereichen einerseits eng an den statistischen Annahmen der Regelsatzbemessung orientiert sein muss und andererseits von der Dauer des Asylbewerberleistungsbezugs abhängt. Nur im Einzelfall könnten auf diese Weise bestimmte abziehbare Positionen identifiziert werden.

Demgegenüber ließe sich der Abzug sämtlicher Kosten für den Bereich Freizeit, Unterhaltung, Kultur nur dann verfassungsrechtlich rechtfertigen, wenn der Gesetzgeber plausibel darlegen kann, warum die Leistungsberechtigten des AsylbLG keinen entsprechenden Bedarf haben. Hierfür müsste der Gesetzgeber die tatsächlichen Lebensverhältnisse der Leistungsberechtigten zugrunde legen und dabei in Rechnung stellen, dass in der Vergangenheit die Leistungen nach dem AsylbLG auf verfassungswidrige Weise unzureichend waren. Es ist daher kaum vorstellbar, dass eine solche empirische Grundlegung gelingen kann.

56 Münder, in: ders. (Hrsg.), Sozialgesetzbuch II, Lehr- und Praxiskommentar, 4. Aufl. 2011, Einl. Rn. 14; s. auch Hebeler, SGb 2008, 8 ff. 


\section{d) Abweichungen etwa für Ausweispapiere}

Der Abzug von Bedarfspositionen des Regelbedarfs des SGB II/SGB XII, für die der leistungsberechtigte Personenkreis des AsylbLG eindeutig keinen Bedarf hat, ist verfassungskonform. Dies gilt etwa für den Abzug der Kosten für den Personalausweis $(0,25 € / \mathrm{Mt}$. Es müssen auch nicht stattdessen Kosten für ausländische Ausweispapiere im Regelsatz berücksichtigt werden. Ihre Nichtbeachtung im Regelsatz rechtfertigt dann, wenn sie bei Bedarf im Einzelfall gewährt werden. Allerdings genügt hierfür eine Ermessensnorm wie $\S 6$ Abs. 1 AsylbLG als Rechtsgrundlage nicht, wenn die Kosten für solche Ausweispapiere als Bestandteil des menschenwürdigen Existenzminimums gewertet werden. Der Gesetzgeber muss sich daher zunächst darüber verständigen, welche Konsequenzen es hätte, wenn mangels finanzieller Mittel solche Ausweispapiere nicht beschafft werden. Auf dieser Grundlage kann der Gesetzgeber sodann entscheiden, ob Kosten hierfür Teil des menschenwürdigen Existenzminimums dieses Personenkreises sind. Gelangt er hier zu einem positiven Ergebnis, ist er von Verfassungs wegen verpflichtet, den Leistungsberechtigten einen bindenden Anspruch auf diese Leistung einzuräumen.

\section{e) Barbetrag für die Bedürfnisse des täglichen Lebens}

Dass der Barbetrag für die Bedürfnisse des täglichen Lebens auf verfassungswidrige Weise zu niedrig ist, hat das Bundesverfassungsgericht festgestellt. ${ }^{57}$ Welche Höhe verfassungsrechtlich geboten ist, ergibt sich daraus, dass sich für diesen Barbetrag keine alternativen Bedarfsermittlungsmethoden erkennen lassen, als die im Rahmen des SGB II/SGB XII praktizierte. Daher sind Abweichungen von der Leistungshöhe nur zulässig, wenn sich hierfür ein sachlich nachvollziehbarer Grund finden lässt.

Jedenfalls verfassungswidrig sind die Pläne, den Barbetrag für Leistungsberechtigte bestimmter Nationalitäten abzusenken, wie dies jüngst von Bundesinnenminister Friedrich vorgeschlagen wurde. Solch eine Binnendifferenzierung unter den Leistungsberechtigten scheitert zum einen daran, dass ihr offensichtlich migrationspolitische Erwägungen zugrunde liegen. Weil der Zuzug aus Serbien und Mazedonien in der letzten Zeit zugenommen hat, soll die Leistungsabsenkung für diese Gruppe Signale gegen einen weiteren Zuzug setzen. Gerade diese Form der Relativierung des menschenwürdigen Existenzminimums zum Zwecke der Migrationssteuerung ist aber vom Bundesverfassungsgericht bereits deutlich zurückgewiesen worden. ${ }^{58} \mathrm{Zudem}$ wird bereits bei kursorischer Betrachtung deutlich, dass eine solche grundrechtsrelevante Leistungsabsenkung Verhältnismäßigkeitsanforderungen nicht genügt. Bei realitätsgerechter Betrachtung erscheint es wenig plausibel, dass die Migrationsbewegungen, die durch die 
europäische Visapolitik entsteht, ${ }^{59}$ durch die Absenkung des Barbetrags im Rahmen des AsylbLG gesteuert werden kann. ${ }^{60}$ Zudem gerät diese Form der Differenzierung in gefährliche Nähe der Diskriminierungsverbote des Art. 3 Abs. 3 GG $^{61}$ - dass sich die „Missbrauchsbefürchtungen“ besonders für die Gruppe der Roma artikulieren, ${ }^{62}$ zeigt dies deutlich - und ist auch mit dem europäischen Diskriminierungsverbot aufgrund der Staatsangehörigkeit (Art. 18 AEUV) nicht vereinbar.

\section{Gewährung des Bildungs- und Teilhabepakets}

Verfassungsrechtlich geboten ist auch die Gewährung der Leistungen für Bildung und Teilhabe. Es gibt keinen sachlichen Grund, diese Leistungen Kindern vorzuenthalten, deren Aufenthaltstitel - regelmäßig aufgrund der Familienakzessorietät - zur Zuweisung zum AsylbLG führt.

Das Bundesverfassungsgericht hat hierzu auch ausdrücklich festgestellt, dass auf Leistungen für Bildung und Teilhabe ein gesetzlicher Anspruch und nicht nur eine Ermessensregelung vorzusehen ist.

\section{Fortschreibung der Leistungen entsprechend den Regelungen des SGB II/SGB XII}

Die verfassungsrechtlich notwendige Fortschreibung der Leistungen darf derjenigen des SGB II/SGB XII entsprechen. Hiergegen bestehen keine verfassungsrechtlichen Einwände.

\section{IV. Änderungen der Zuordnung zum AsylbLG}

Die skizzierte gegenwärtige Reformdiskussion wird im Wesentlichen um die Frage der richtigen Leistungshöhe geführt. Dies beruht auf der Fragestellung und damit dem Gegenstand des Urteils des Bundesverfassungsgerichts. Denn es hat die Leistungshöhe des

59 Seit Dez. 2009 besteht für Staatsangehörige von Mazedonien, Montenegro und Serbien keine Visumspflicht mehr im Schengenraum, wenn sie über biometrische Pässe verfügen, vgl.: Anhang II, Teil 1 der EU-VisaVO. Seit 2008 steigt die Zahl der Asylbewerber kontinuierlich. Im Oktober 2012 wurde nun ein starker Anstieg der Asylerstanträge festgestellt. Hiervon stellen Personen aus Serbien und Mazedonien die stärksten Gruppen; vgl.: Bundesamt für Migration und Flüchtlinge, Aktuelle Zahlen zu Asyl, Ausgabe Oktober 2012, http://www.ba $\mathrm{mf}$.de/SharedDocs/Anlagen/DE/Downloads/Infothek/Statistik/statistik-anlage-teil-4-aktuell e-zahlen-zu-asyl.pdf;jsessionid=E0ADD7CEEF0F70E8E8A5EC216C9FAA3E.1_cid244? blob=publicationFile (abgerufen 8.12.2012).

60 Die Wiedereinführung der Visumspflicht wird daher gefordert: http://www.bild.de/politik/in land/hans-peter-friedrich/innenminister-klagt-ueber-fluechtlings-ansturm-auf-deutschland-2 6683892.bild.html (abgerufen am 7.12.12).

61 Zur Differenzierung nach der Staatsangehörigkeit vgl. Wallrabenstein, Staatsangehörigkeit Differenzierungsgrund wofür?, in: Barwig/Beichel-Benedetti/Brinkmann, Gleichheit - Hohenheimer Tage zum Ausländerrecht 2011, 2012, S. $416 \mathrm{ff}$.

$62 \mathrm{http}: / / \mathrm{www}$. fr-online.de/politik/steigende-asyl-antraege-friedrich-will-roma-geld-verweigern-,1472596,20706638.html (abgerufen am 7.12.12). 
$\S 3$ AsylbLG für verfassungswidrig erklärt. Systematisch stehen aber eigentlich andere Fragen im Vordergrund, auf die deshalb abschließend eingegangen werden soll.

\section{Ist eine Überführung in das SGB II/SGB XII verfassungsrechtlich geboten?}

In der rechtspolitischen Diskussion ist bisher meist nur zwischen der abgesenkten Leistungshöhe nach $\S 3$ AsylbLG und den sogenannten Analogleistungen nach $\S 2$ AsylbLG unterschieden worden. Wenig erörtert ist bisher die Frage, ob diese sog. Analogleistungen selbst verfassungsrechtlichen Anforderungen genügen oder ob nicht Zugang zu den Leistungen des SGB II notwendig ist.

Die Rechtsprechung des Bundessozialgerichts, die keine verfassungsrechtlichen Bedenken an der dauerhaften Zuweisung der Personengruppen des § 1 Abs. 1 AsylbLG zu einem Sondersystem hatte, ${ }^{63}$ lässt sich aber angesichts des Urteils des Bundesverfassungsgerichts vom 18.7.2012 nicht mehr aufrechterhalten. Das Bundessozialgericht hatte den legitimen Differenzierungsgrund zum einen in der fehlenden Aufenthaltsperspektive und zum anderen in dem gesetzgeberischen Ziel gesehen, Migrationsanreize $\mathrm{zu}$ verhindern. Es wurde oben ${ }^{64}$ herausgearbeitet, dass die bisherige Regelung die gebotene kurze Aufenthaltsperspektive der Leistungsberechtigten nicht sichergestellt hat. Auch ist das Verhindern von Migrations- bzw. Bleibeanreizen kein zulässiges Ziel, das die Relativierung des menschenwürdigen Existenzminimums rechtfertigen könnte. ${ }^{65}$ Damit entfallen aber bereits die Kriterien, die die Sonderung der Leistungsberechtigten für die abgesenkten ,eigentlichen“ Leistungen des AsylbLG rechtfertigen sollten. Noch weniger können sie begründen, warum Personen, die gar nicht die abgesenkten Leistungen erhalten sollen, sondern im Ergebnis gerade wieder weitgehend anderen Hilfebedürftigen gleichgestellt werden, in diesem Sondersystem verbleiben sollen.

Es ist auch kein sachlicher Grund dafür erkennbar, warum Ausländergruppen, die zwar ausreisen sollten aber nicht können, und die in Deutschland erwerbstätig sein dürfen und erwerbstätig sein sollen, anders behandelt werden als im Leistungssystem des SGB II.

Der Mangel sachlicher Differenzierungsgründe führt allerdings erst dann zu einem Verfassungsverstoß, wenn mit der Zuordnung zum AsylbLG anstelle des SGB II/ SGB XII grundrechtsrelevante Unterschiede ergeben. Werden die Leistungen - als Analogleistungen - in gleicher Höhe und in gleichem Umfang gewährt, so besteht kein grundrechtsrelevanter Unterschied. Systematische Gründe mögen gegen eine solche Lösung sprechen, aber sie allein führen noch nicht zur Verfassungswidrigkeit.

63 S.o.: BSGE 102, 60.

64 Hierzu s.o. I. 1. a.

65 S.o. 


\section{Auf welchen Personenkreis kann/darf sich das AsylbLG beziehen?}

Soll aber für eine bestimmte Zeit (dazu sogleich) ein bestimmter Personenkreis einem anderen Leistungssystem zugeordnet werden, so muss der Gesetzgeber mit Sorgfalt prüfen, welche Sachgründe hierfür bestehen. ${ }^{66}$

Sachlich nachvollziehbar ist die Zuordnung zum Asylbewerberleistungsgesetz mit seinem Konzept abgesenkter und andersartiger Leistungen - wie bereits der Name nahelegt - für Asylbewerber und Flüchtlinge mit Aufenthaltstitel nach $\S 23$ AufenthG unmittelbar nach der Einreise. Für Personen, die erst deutlich nach ihrer Einreise, womöglich auch nicht mehr in zeitlichem und sachlichem Zusammenhang zur Einreise Asyl beantragen, lässt sich diese Zuweisung schon nicht mehr begründen.

Warum Personen mit anderen Aufenthaltstiteln, insbesondere den Titeln nach $\S 25$ Abs. 4 bis 5 AufenthG und mit einer Duldung, ebenfalls dem AsylbLG zugewiesen werden sollen, ist nur schwer begründbar. Diese Aufenthaltstitel und die Duldung werden regelmäßig nicht gleichzeitig mit der Einreise erteilt, sondern schließen sich an andere Titel oder Zeiten des Aufenthalts ohne Aufenthaltstitel an. Es ist nicht erkennbar, warum diese Personen in Bezug auf ihren Existenzbedarf mit denen vergleichbar sind, die soeben erst nach Deutschland eingereist sind.

Nachvollziehbar ist nur die Einbeziehung von den Personen, die zunächst eine Aufenthaltsgestattung gemäß $\S 55$ AsylVerfG besaßen und sich an diese Gestattung (nach Ablehnung des Asylgesuchs) einer der genannten Aufenthaltstitel oder die Duldung unmittelbar anschließt. Sollen etwa die besonderen Leistungen nach dem AsylbLG für ein Jahr gewährt werden, wird das Asylverfahren in einem Beispielsfall nach drei Monaten erfolglos abgeschlossen und es stehen der Ausreise Hindernisse entgegen, die so die Erwartung - innerhalb der nächsten Monate ausgeräumt werden können, so ist es sachlich nachvollziehbar, dass diese Person trotz Wechsel des Aufenthaltstitels bzw. der Duldung weiterhin dem AsylbLG zugeordnet bleibt. Zu empfehlen ist daher eine solche Beschränkung des Personenkreises.

\section{Welche Dauer kann der als Leitbild für das AsylbLG dienende Kurzaufenthalt ha- ben?}

Schließlich ist die Dauer der Zuordnung zum AsylbLG - bzw. zu den abgesenkten Leistungen gemäß $\S 3$ AsylbLG - zentral. Oben wurde ausgeführt, dass eine abweichende Leistungsbestimmung umso schwerer wird, je länger die zugrunde gelegte Aufenthaltsdauer ist. Der Gesetzgeber muss sich deshalb zunächst darüber klar werden, welchen Bezugszeitrahmen er regeln will. Sinnvollerweise sollte er sich dabei an Fristen anderer thematisch einschlägiger Regelungen orientieren.

Das Bundesverfassungsgericht hat ausdrücklich darauf hingewiesen, dass ein abweichendes Existenzminimum bestimmter Ausländergruppen nur dann verfassungsrechtlichen Anforderungen genügen kann, wenn es nur für die Zeit eines Kurzaufenthaltes gilt.

Ein möglicher Anknüpfungspunkt wäre daher eine Aufenthaltsdauer von bis zu drei Monaten. Damit würde der Kurzaufenthalt, der für das AsylbLG maßgeblich ist, dem

66 S.o. insb. zu Art. 3 (unter II. 2.). 
Kurzaufenthalt nach dem sog. Schengenrecht entsprechen. Die Bezugsdauer nach dem AsylbLG wäre deckungsgleich mit der maximalen Zeit in Erstaufnahmeeinrichtungen. Da unter diesen Bedingungen der Unterschied zu „normalen“ Leistungsberechtigten nach dem SGB II/SGB XII offensichtlich ist, fiele es nicht schwer, abweichende Regelungen und abweichende Bedarfe zu begründen.

Ein anderer Anknüpfungspunkt kann etwa die Möglichkeit zur Erwerbstätigkeit sein. Asylbewerber dürfen nach einem Jahr Aufenthalt in Deutschland erwerbstätig sein, wenn auch unter Vorbehalt der Vorrangprüfung gemäß § 39 AufenthG. Es wäre sachlich nachvollziehbar, wenn sich der Gesetzgeber bei den Leistungen nach dem AsylbLG auf den Zeitraum beschränkte, in dem Erwerbstätigkeit grundsätzlich nicht erlaubt ist. In dieser Zeit besteht eine höhere Wahrscheinlichkeit, dass die Betroffenen ihr Existenzminimum erst durch staatliche Leistungen decken können. Außerdem ist es plausibel anzunehmen, dass sich die Bedarfe von Personen, die erwerbstätig sein können, soweit ähneln, dass sie auch bei den Hilfeleistungen grundsätzlich gleich zu behandeln sind. Die Entwicklung eines besonderen Leistungssystems mit abweichenden Bedarfen wird daher für die Personengruppe mit Aufenthalt von einem Jahr und mehr kaum möglich sein. Bis zu einem Jahr scheint eine Differenzierung aber noch nachvollziehbar. Freilich wären die Begründungen der konkreten Leistungsabsenkungen für diesen längeren Zeitraum bereits schwieriger. ${ }^{67}$

\section{Fazit}

Die Entwicklung des AsylbLG - bzw. seine fehlende Weiterentwicklung in den vergangenen 20 Jahren - haben eine Entscheidung des Bundesverfassungsgerichts geradezu überfällig gemacht. In entsprechender Eindeutigkeit ist das Urteil vom 18.7.2012 ausgefallen.

Irritationen wirft allerdings die gegenwärtige - zudem verhaltene-Reformdiskussion auf. Während wichtige Leistungsverbesserungen, insbesondere die grundsätzliche Orientierung an der Regelsatzbemessung in der Grundsicherung, ein gesetzlicher Anpassungsmechanismus und die gesetzliche Zuerkennung der Leistungen des Bildungs- und Teilhabepaket zwingend sind und unstreitig scheinen, bestehen doch sehr unterschiedliche Ideen, wie und in welchem Umfang eine Leistungsabsenkung, teilweise verbunden mit entsprechender Sachleistungsgewährung, möglich sind. Hier wurde aufgezeigt, dass der Gestaltungsspielraum des Gesetzgebers hierbei ernst zu nehmen ist, er aber bei dessen Nutzung eine erhebliche Argumentationslast zu schultern hat. Dass migrationspolitische Erwägungen hierbei keinen Platz haben, scheint sich - erschreckender Weise noch nicht bis zu allen politisch Verantwortlichen herumgesprochen zu haben.

Bedauerlich ist, dass bei den Reformüberlegungen die zentrale Frage, welche Personengruppen und wie lange einem Sondersystem zugeordnet werden sollen, kaum oder nur untergeordnet diskutiert wird. Dabei werden hier auch die Weichen für die Legitimation der Leistungsdifferenzierung gestellt.

Daher erscheinen die Vorschläge, das AsylbLG insgesamt abzuschaffen, womöglich am ehesten praktikabel. Es wäre einer handlungsfähigen Politik zu wünschen, dass sie

67 Hierzu s.o. II 1 d. 
nicht so lange komplizierte Lösungen sucht, bis letzten Endes ein weiteres Mal das Bundesverfassungsgericht auf den Plan gerufen wird und womöglich genau dieses Ergebnis herbeiführt. Vorstellbar wäre es. 